\title{
Direct Numerical Simulations of Autoignition in Stratified Dimethyl-ether (DME)/Air Turbulent Mixtures
}

\author{
Gaurav Bansal ${ }^{\mathrm{a}, 1, *}$, Ajith Mascarenhas ${ }^{\mathrm{a}, 2}$, Jacqueline H. Chen ${ }^{\mathrm{a}}$ \\ ${ }^{a}$ Combustion Research Facility, Sandia National Laboratories, Livermore, CA - 94551
}

\begin{abstract}
In this paper, two- and three-dimensional direct numerical simulations (DNS) of autoignition phenomena in stratified dimethyl-ether (DME)/air turbulent mixtures are performed. A reduced DME oxidation mechanism, which was obtained using rigorous mathematical reduction and stiffness removal procedure from a detailed DME mechanism with 55 species, is used in the present DNS. The reduced DME mechanism consists of 30 chemical species. This study investigates the fundamental aspects of turbulence-mixing-autoignition interaction occurring in homogeneous charge compression ignition ( $\mathrm{HCCI}$ ) engine environments. A homogeneous isotropic turbulence spectrum is used to initialize the velocity field in the domain. The computational configuration corresponds to a constant volume combustion vessel with inert mass source terms added to the governing equations to mimic the pressure rise due to piston motion, as present in practical engines. DME autoignition is found to be a complex three-staged process; each stage cor-
\end{abstract}

\footnotetext{
${ }^{*}$ Corresponding author. Ph: +1-734-239-2516, Address: 2111 NE 25th Ave, Hillsboro, OR 97124, USA

Email address: gaurav2. bansal@intel.com (Gaurav Bansal)

${ }^{1}$ Present Affiliation: Intel Corporation, Hillsboro, OR, USA

${ }^{2}$ Present Affiliation: Optimizely, San Fransisco, CA, USA
} 
responds to a distinct chemical kinetic pathway. The distinct role of turbulence and reaction in generating scalar gradients and hence promoting molecular transport processes are investigated. By applying numerical diagnostic techniques, the different heat release modes present in the igniting mixture are identified. In particular, the contribution of homogeneous autoignition, spontaneous ignition front propagation, and premixed deflagration towards the total heat release are quantified.

Keywords: low temperature combustion engines, thermal and compositional stratification, autoignition, direct numerical simulation.

\section{Nomenclature}

$\gamma \quad$ Ratio of specific heats

$\nu \quad$ Kinematic viscosity of air

$\bar{\phi} \quad$ Mean equivalence ratio

$\bar{\rho} \quad$ Mean density

$\bar{T} \quad$ Mean temperature

c Speed of sound

$L \quad$ Physical domain length

$L_{11, T}$ Autocorrelation integral length scale of temperature fluctuations

$L_{11, u}$ Autocorrelation integral length scale of velocity fluctuations

$L_{11, Z}$ Autocorrelation integral length scale of mixture fraction fluctuations

$N$ Number of grid points

$P$ Pressure

$R \quad$ Specific gas constant

$R e$ Turbulent reynolds number based on integral length scale 
$T^{\prime}$ Temperature RMS

$u^{\prime} \quad$ Velocity RMS

$Z^{\prime}$ Mixture fraction RMS

\section{Introduction}

Homogeneous charge compression ignition (HCCI) is a viable new concept for next-generation internal combustion engines [1]. It has the potential for achieving ultra-low nitric oxides (NOx) and soot emissions while concurrently providing higher fuel conversion efficiency. Conventionally, HCCI refers to a combustion regime wherein the charge is homogeneous in terms of temperature and mixture composition, in which combustion occurs primarily via volumetric autoignition. The premixed mixture is extremely fuel-lean which results in a low burn temperature, and therefore, formation of NOx and soot is mitigated. Two major technical impediments to the realization of the HCCI concept are the high rates of pressure rise since the entire charge ignites homogeneously, and ignition control and combustion phasing control which becomes difficult resulting in large cycle-to-cycle variations. To alleviate these issues, some degree of charge stratification is deliberately introduced $[2,3]$ in order to avoid extremely rapid pressure rise and heat release rates in the engine. Exhaust gas recirculation [4] and multiple fuel injection [5] are some of the techniques that are employed to introduce charge stratification in the engine cylinder. Moreover, some thermal stratification instrinsically exists in the cylinder due to wall heat loss. Due to the presence of turbulence, large scale stratification of charge leads to small scale inhomogeneities in both temperature and fuel mass fraction. In view of these control strategies, it is essential to thoroughly investigate the autoignition and subsequent combustion behav- 
ior in high pressure stratified turbulent mixtures. A fundamental understanding of turbulence-mixing-autoignition interaction in stratified systems will provide some of the necessary insights to aid in the optimal design of these engines.

Furthermore, a variety of fuel options are currently being studied for powering HCCI engines. Recent research has investigated the use of various blends of conventional gasoline and diesel fuels with bio-derived alcohols and ethers [6, 7]. In the present study, we investigate the combustion characteristics of dimethyl ether (DME) in HCCI engine environments. DME can be synthetically bio-dervied from lignocellulosic biomass, and is a promising alternative fuel for HCCI engines owing to its favorable autoignition properties. It has a cetane number of 55 , larger than the cetane number for diesel (44-53). It can lead to reduced emissions of soot and NOx, compared to conventional diesel fuels [8]. The soot reduction is a direct result of the chemical structure of DME, i.e. it doesn't have a C-C bond to act as a seed for polymerization which leads to generation of polyaromatic hydrocarbons. And since DME can be burned over a wide range of equivalence ratios without forming soot, it is easy to optimize the combustion system with the use of after-treatment strategies to generate low NOx.

Yamada et al. [9] elucidated the chemical mechanism of DME oxidation by using an externally motored single-cylinder piston engine. It was shown using insitu laser-induced fluorescence that formaldehyde forms rapidly during the firststage ignition process, corresponding to cool flame ignition, and subsequently disappears during the second-stage of thermal ignition. This is consistent with the detailed chemical mechanism of DME oxidation by Curran et al. [10]. Yamada et al. [9] also developed a simple set of chemical reactions with limited rate parameters which could adequately represent the heat release from cool ignition. 
More recently, Yamada et al. [11] experimentally investigated the transition from cool flame to thermal explosion in DME-fired HCCI engines. They concluded that the mechanism during this transition process can be qualitatively explained using the thermal explosion theory, whereby the rate-determining reaction is $\mathrm{H}_{2} \mathrm{O}_{2}$ decomposition, assuming that the heat release in this period is caused by partial oxidation of fuel and intermediate species. Moreover, they clearly showed that DME autoignition can be partitioned into three distinct ignition stages, each attributed to a different chemical reaction pathway. The first stage corresponds to the conventional cool ignition (negative-temperature coefficient (NTC) regime) controlled by DME fuel-specific chemistry. The key-intermediate in this low-temperature ignition process was found to be methoxymethyl-peroxy $\left(\mathrm{CH}_{3} \mathrm{OCH}_{2} \mathrm{O}_{2}\right)[9,12]$. The second stage is controlled by $\mathrm{H}_{2} \mathrm{O}_{2}$ thermal dissociation, and the final stage corresponds to high temperature ignition of hydrogen controlled by the well known branching-termination reaction pair:

$$
\begin{gathered}
\mathrm{H}+\mathrm{O}_{2} \rightarrow \mathrm{O}+\mathrm{OH} \\
\mathrm{H}+\mathrm{O}_{2}+\mathrm{M} \rightarrow \mathrm{HO}_{2}+\mathrm{M} .
\end{gathered}
$$

The three-staged DME autoignition is unique compared to other fuels with NTC chemistry such as $n$-heptane due to the presence of a visible and separated heat release peak for $\mathrm{H}_{2} \mathrm{O}_{2}$ thermal dissociation (second stage of DME autoignition).

In light of the aforementioned discussion, the goal of the present study is to use high-fidelity direct numerical simulations to investigate the autoignition and subsequent combustion behavior in high-pressure turbulent stratified DME/air mixtures. The computational configuration corresponds to a three-dimensional constant volume cube, with the turbulent velocity field initialized using a turbulence 
energy spectrum. Inhomogeneous temperature and fuel mass fraction fields are initialized by incorporating insights gained from optical engine experiments performed elsewhere $[13,14]$. In practical engines, the low-temperature heat release phenomenon generally occurs when the piston is moving up in the compression stroke. Inert mass addition source terms are added to the governing equations in the DNS to mimic this isentropic compression effect due to piston motion. A few two-dimensional DNS runs are first performed to identify the parameter space for which a three-dimensional DNS is subsequently performed.

A number of studies in the last decade have investigated autoignition phenomena in a similar high-pressure constant volume configuration $[15,16,17,18$, $19,20,21,22,23,24]$. These studies have provided physical insights into the mechanism of autoignition in stratified turbulent mixtures. In particular, it has been demonstrated in these studies and also in a variety of experimental studies $[25,26,27,28,29]$ that autoignition in stratified systems can lead to a variety of heat release modes such as homogeneous autoignition, spontaneous ignition front propagation [30], and premixed deflagration. These studies have also conducted parametric investigation of various key variables such as stratification magnitude, turbulence intensities, initial correlation between thermal and compositional stratification, etc. and their effects on ignition delay and heat release modes. Another recent study investigated the ignition and flame regimes in $n$-heptane-air mixtures in spark-assisted HCCI conditions using a one-dimensional configuration [31]. Given the body of existing literature, the novelty and objectives of the present study are the following:

- DME chemistry. Apart from Shreedhara and Lakshmisha [15], who used a simple four-step $n$-heptane chemistry, and a few very recent studies [20, 24, 
$22,23]$, all the other aforementioned computational studies used hydrogen as the fuel. Although hydrogen is important in its own right as a potential fuel for HCCI engines and it also forms a building block for more complex hydrocarbon fuel chemistry, it does not contain the NTC regime prevalent in some practical engine fuels. The present study incorporates a reduced DME chemical mechanism [32], systematically reduced and validated from a detailed mechanism [12], through rigorous mathematical techniques. DME is a potential fuel for HCCI engines and it also exhibits NTC chemistry. As such, it offers a richer investigation of turbulence-mixing-autoignition interaction. El-Asrag and Ju recently studied DME autoignition in a similar constant volume configuration $[22,23]$. They investigated the effects of exhaust gas recirculation of autoignition using $\mathrm{H}_{2} \mathrm{O}_{2}$ as a representative EGR species [22]. They also found that molecular diffusion plays an important role in the autoignition process [22]. In [23], El-Asrag and Ju study the effects of $\mathrm{NO}_{\mathrm{x}}$ on DME autoignition. It is shown that DME autoignition occurs in three distinct stages, which has also been showed by other experimental studies [11] and is also demonstrated in the present study. They used three different criterions based on: mixing scalar dissipation rates, a Damköhler number defined as a ratio of mixing and chemical times scales, and displacement speed, to characterize the nature of the flame propagation modes. The present work further investigates the DME autoignition through an extensive parametric study and reveals some interesting new findings.

- Three-dimensional turbulence. Although 2-D simulations do provide significant insights into the phenomena of interest, turbulence is inherently three-dimensional. Therefore, from a modeling perspective, it is desirable 
to understand turbulence-autoignition interactions in a 3-D turbulence field. Apart from Shreedhara and Lakshmisha [15], all the other studies noted above investigated two-dimensional turbulence. To the authors' knowledge, the present study is the first to investigate autoignition in the abovementioned configuration incorporating both a detailed description of chemical kinetics as well as three-dimensional turbulence. Supercomputers have only very recently become powerful enough to undertake such a massive task. The 3-D simulation presented in this study required approximately 10 Million compute-hours on Jaguar, a Cray XT5 system at Oak Ridge National Laboratories.

- Isentropic compression. Another novelty of the present work is the incorporation of inert mass (density) source terms in the governing equations to reproduce the compression resulting from piston motion. This is essential as low-temperature heat release usually occurs during the compression stroke in practical engines. Due to the initial thermal stratification, the specific heat is also non-homogeneous and, therefore, the isentropic compression also results in modification of thermal gradients prior to heat release. A detailed derviation of the local density source term is given in Appendix A.

The paper is organized as follows. Section 2 presents the details of the numerical implementation and initial conditions used for the DNS. Sections 3, 4 and 5 present zero-, two- and three-dimensional DNS results, respectively. Finally, Section 6 summarizes the key findings of the present study. 


\section{Numerical methodology and initial conditions for DNS}

In this section, the solution algorithm and initial conditions for the DNS are presented. The full compressible Navier-Stokes, species, and energy equations for a reacting gas mixture are solved using the Sandia DNS code, S3D. The code employs a fourth-order Runge-Kutta method for time integration and an eighthorder explicit spatial difference scheme [33, 34]. CHEMKIN [35] and TRANSPORT [36] libraries are linked to S3D to evaluate reaction rates, thermodynamic and mixture-averaged transport properties. A reduced chemical mechanism for DME oxidation consisting of 30 chemical species is employed in this study [32].

Table 1: Initial mean mixture conditions

\begin{tabular}{cc} 
& $677.73 \mathrm{~K}$ \\
$\bar{T}$ & \\
$\bar{\phi}$ & 0.6 (cases b-2D and c-2D); 0.3 (all other cases) \\
$P$ & $10.834 \mathrm{~atm}$ \\
\hline
\end{tabular}

Table 2: Isentropic compression parameters

\begin{tabular}{cc}
\hline Compression ratio & 18 \\
Ratio of connecting rod length to crank-shaft length & 3.2 \\
Engine RPM & 1200 \\
Initial crank angle & $324^{\circ}$ BTDC \\
\hline
\end{tabular}

The computational domain consists of a square (for 2-D cases) and a cube (for the 3-D case). Periodic boundary conditions are employed at all of the boundaries. 
Table 3: Numerical and turbulence parameters for the different cases

\begin{tabular}{cccccccccc}
\hline Case & $u^{\prime}$ & $L_{11, u}$ & $T^{\prime}$ & $L_{11, T}$ & $Z^{\prime}$ & $\begin{array}{c}L_{11, Z} \\
(m m)\end{array}$ & $\begin{array}{c}\mathrm{Re} \\
\left(=u^{\prime} L_{11, u} / \nu\right)\end{array}$ & $\begin{array}{c}L \\
(m m)\end{array}$ \\
\hline a-NR-2D & 0.375 & 0.375 & 25 & 0.375 & 0.0 & $\mathrm{NA}$ & 24 & 3.2 & 560 \\
b-NR-2D & 0.375 & 0.375 & 25 & 0.375 & 0.0 & $\mathrm{NA}$ & 24 & 3.2 & 560 \\
a-2D & 0.5 & 0.5 & 25 & 0.5 & 0.0 & $\mathrm{NA}$ & 43 & 4 & 560 \\
b-2D & 0.5 & 0.5 & 25 & 0.5 & 0.006 & 0.5 & 43 & 4 & 1200 \\
c-2D & 0.5 & 0.5 & 25 & 0.5 & 0.006 & 0.5 & 43 & 4 & 1200 \\
d-2D & 0.0 & $\mathrm{NA}$ & 25 & 0.375 & 0.0 & $\mathrm{NA}$ & 0.0 & 3.2 & 560 \\
e-2D & 0.375 & 0.375 & 25 & 0.375 & 0.0 & NA & 24 & 3.2 & 560 \\
a-3D & 0.375 & 0.375 & 25 & 0.375 & 0.0 & NA & 24 & 3.2 & 560 \\
\hline
\end{tabular}


As mentioned in the Introduction, inert mass addition source terms are added to the governing equations to mimic the pressure rise due to piston motion in practical engines. These are local density source terms for which a derivation is given in Appendix A. Contributions of the density source term appear in the continuity equation, and consequently, in the momentum, energy, and species conservation equations.

The initial mean conditions for all of the cases investigated in this study are given in Table 1. Apart from two cases, all other cases simulated are only thermally stratified and have an initial equivalence ratio of 0.3 ; the cases with both thermal and equivalence ratio stratification have an initial mean equivalence ratio of 0.6. The engine parameters used to evaluate the change in volume (via the inert mass source terms) for all cases are given in Table 2. A suite of 2-D cases and one 3-D case are investigated in the present study. A description of these cases will be given in the rest of the paper. The various turbulence parameters and numerical grid parameters used for these cases are given in Table 3. The spatial resolution requirements for all these cases stems from the need to resolve the thinnest radical and reaction rate layers in the combustion front. For this purpose, a series of 1-D simulations are performed with different grid resolutions to inform the multi-dimensional DNS of the required resolution.

The mean flow for all cases is quiescent initially. At the initial time, turbulence velocity fluctuations were superimposed on the quiescent mean velocity field based on the Passot-Pouquet turbulent kinetic energy spectrum function [37]:

$$
E(k)=C_{s} \sqrt{\frac{2}{\pi}} \frac{u^{\prime 2}}{k_{e}}\left(\frac{k}{k_{e}}\right)^{4} \exp \left[-2\left(\frac{k}{k_{e}}\right)^{2}\right]
$$

where $C_{s}=32 / 3$ for $2 \mathrm{D}$, and 16 for $3 \mathrm{D}$ cases. Random temperature and compo- 
sition fields were superimposed on the mean temperature and composition fields, respectively. The temperature and equivalence ratio spectrums are similar to the turbulence kinetic energy spectrum and are used to specify the characteristic scales of initial hot/cold spots and initial rich/lean fuel pockets, respectively. First, two non-reacting cases were simulated to investigate the effects of isentropic compression due to piston motion (here simulated via inert mass source terms) on scalar mixing. Case a-NR-2D is a non-reacting case in which the compression source terms were added to the governing equations. Case b-NR-2D represents a nonreacting case with no compression source terms. All other cases in Table 3 correspond to reacting cases. Apart from cases b-2D and c-2D which were initialized with inhomogeneities in both temperature and equivalence ratio, all other cases were initialized with only temperature stratification. The typical initial temperature and vorticity fields (shown here for the 3-D case, a-3D) are shown in Figs. 1 and 2, respectively. Figure 1 shows the superposition of hot and cold pockets of different length-scales on the mean temperature field. Fig 2 shows that the vorticity is concentrated in tube- and platelet-like structures. Case b-2D corresponds to an initially uncorrelated $T-\phi$ field, whereas case c-2D corresponds to an initially negatively-correlated $T-\phi$ field. $T$ varies independently of $\phi$ in the uncorrelated $T-\phi$ case, whereas $T$ is high where $\phi$ is low and vice-verca in the negativelycorrelated $T-\phi$ case. As discussed in Ref. [21], the two limiting scenarios might exist in practical engines depending upon parameters such as the start of injection timing, wall heat loss, stratification in EGR, and in-cylinder turbulence. In case $\mathrm{d}-2 \mathrm{D}$ there is no initial turbulence, and therefore, all mixing occurs solely due to molecular transport. A comparison of cases d-2D and e-2D will identify whether turbulence plays a major role in these systems. 


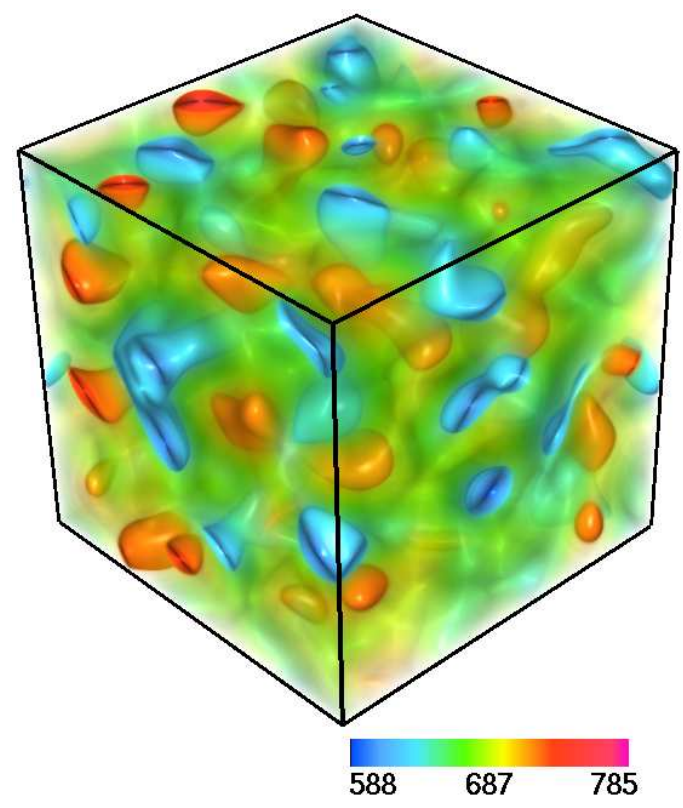

Figure 1: Initial temperature field $(\mathrm{K})$ for the 3D case, case a-3D.

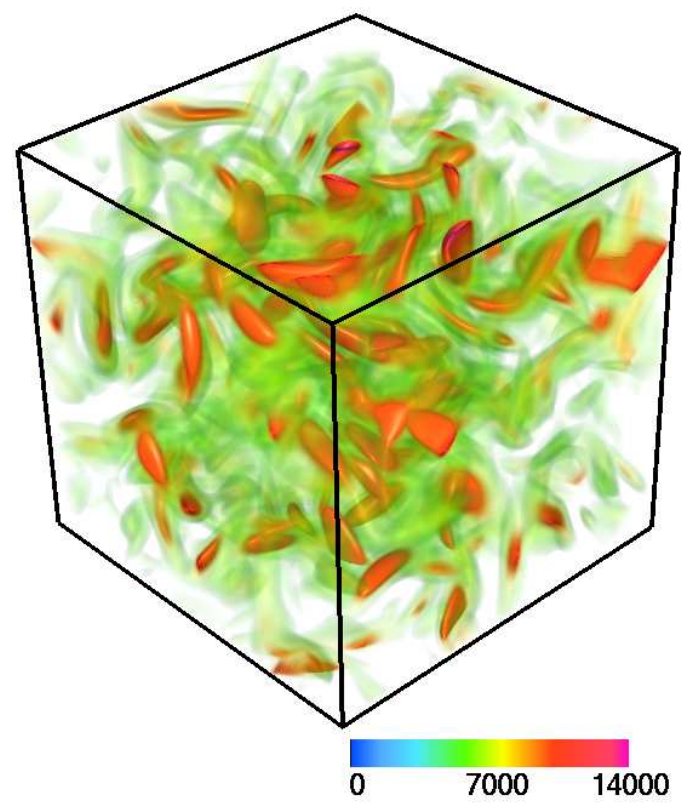

Figure 2: Initial vorticity field $\left(\mathrm{sec}^{-1}\right)$ for the 3D case, case a-3D. 
The initial autocorrelation integral length scale of the velocity $\left(L_{11, u}\right)$ and scalar fluctuations $\left(L_{11, T}\right)$ is determined by integrating the prescribed energy spectrum. The values of this scale for the various cases is given in Table 3. The integral scale Reynolds number $(R e)$ based on this scale is also given in Table 3 . To calculate $R e$, the kinematic viscoisty of air at the initial mean conditions given in Table 1 is used ( $\nu=5.88 \mathrm{e}-6 \mathrm{~m}^{2} / \mathrm{s}$ at $10.834 \mathrm{~atm}$ and $\left.677.73 \mathrm{~K}\right)$. As seen in Table 3 the turbulence integral time scale $\left(\tau_{t}=u^{\prime} / L_{11, u}\right)$ and $T^{\prime}$ for all cases is fixed to $1 \mathrm{~ms}$ and $25 \mathrm{~K}$, respectively. In a typical engine, $u^{\prime}$ values are found to be of the order of $5 \mathrm{~m} / \mathrm{s}$, and $L_{11, \mathrm{u}}$ is of the order of $6 \mathrm{~mm}$ [13]. This leads to a turbulent integral time scale of $1.2 \mathrm{~ms}$ and an integral scale Reynolds number of 9000 for a typical engine. Moreover, in a recent experimental study performed in an optical engine [14], the temperature fluctuation RMS in an HCCI engine was found to be $13.3 \mathrm{~K}$ at top-dead center (TDC), which is of the same order as employed in the present DNS $\left(T^{\prime}=25 \mathrm{~K}\right)$. Thus the integral time scale and $T^{\prime}$ employed in the present DNS are comparable to those in a real engine. As discussed in Ref. [21], matching the turbulence integral time scale and $T^{\prime}$ leads to temperature gradients which are comparable in DNS and real engines. This is the case even though the initial length scales of inhomogeneities $\left(L_{11, T}\right)$ are much larger in real engines than those specified in the present DNS study.

In a previous study [17], the local temperature gradient was identified to be the key parameter affecting molecular transport in high heat release regions. Thus, as demonstrated by Hawkes et al. [19], a change in initial length scales may not affect the heat release behavior as long as the turbulence integral time $\operatorname{scale}\left(\tau_{t}\right)$ and $T^{\prime}$ are kept fixed. Therefore, the heat release behavior observed in the present DNS study is expected to be relevant in a real engine. It should be noted that 
for the cases in which mixture concentration gradient is present, this also plays a part in affecting the molecular transport in high heat release regions; the role of mixture concentration gradient is analogous to that of thermal gradients, when appropriately normalized. Furthermore, the homogeneous ignition delay time for DME autoignition computed at the mean temperature and mean equivalence ratio $(=0.3)$ employed in the DNS is found to be $2.46 \mathrm{~ms}$, which is also comparable to ignition delay times of realistic fuels in a typical engine. The larger Reynolds number present in the engine will primarily contribute towards producing a larger range of scales; however, the finer scale turbulence-chemistry interactions observed in DNS are expected to be similar to those present in real engines.

\section{Zero-dimensional simulations}

In this section, the results of zero-dimensional (homogeneous) constant volume ignition results for DME autoignition are presented. These results provide a reference for the 2-D and 3-D cases presented in subsequent sections, and also provide a chemical description of DME autoignition without the additional complexities of molecular and turbulent transport. Fig. 3 shows the ignition delay $\left(\tau_{\text {ign }}\right)$ as a function of initial temperature $\left(T_{o}\right)$. Here, the definition of ignition delay is based on the time corresponding to the maximum rate of temperature rise during the third (or final) stage of ignition. The initial equivalence ratio and pressure for all cases is 0.3 and 27.22 atm, respectively. A typical negative-temperature coefficient (NTC) regime is observed in the ignition delay plot. As previously mentioned, the 2-D and 3-D cases to be presented in the following sections were performed with isentropic compression (via inert mass source terms), and thus the temperature and pressure of the system rises uniformly in time. The range $\Delta T_{o}$ 
shown in Fig. 3 corresponds to the range of initial temperature distribution for an equivalent case (b-NR-2D) excluding isentropic compression. At the limits of this range $\left(T_{o}=743.5 \mathrm{~K}, 928.5 \mathrm{~K}\right)$ the evolution of temperature and heat release rate (HRR) is given in Fig. 4. The two limiting temperatures show a very different time history. The low temperature $\left(T_{o}=743.5 \mathrm{~K}\right)$ case exhibits three peaks in HRR with roughly equal magnitude, and there is a corresponding distinct rise in temperature in each of these three stages. On the other hand, for the high temperature case ( $T_{o}=928.5 \mathrm{~K}$ ) the low temperature heat release (first ignition stage) is negligible compared to the other two stages. The third-stage results in the maximum HRR and a corresponding temperature rise in this case. As will be presented in the following sections, interesting dynamics exist in 2-D and 3-D simulations where the initial temperature distribution corresponds to a range of values between these two limiting cases.

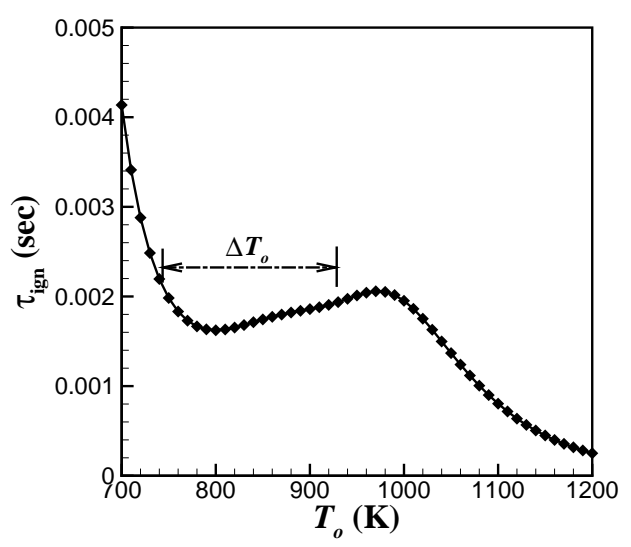

Figure 3: Ignition delay as a function of initial temperature for a zero-dimensional reactor at $\phi=$ 0.3 and $p=27.22$ atm.

As a reference for the case with isentropic compression, Fig. 5 shows the tem- 


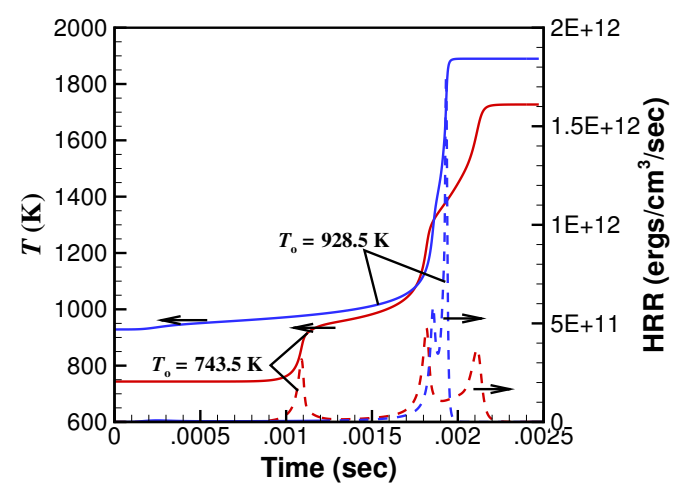

Figure 4: Evolution of temperature and heat release rate (HRR) for two different initial temperatures, for a zero-dimensional case at $\phi=0.3$ and $p=27.22 \mathrm{~atm}$.

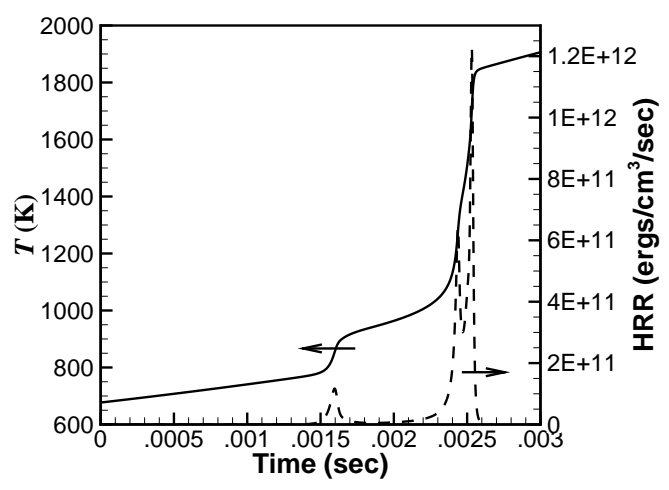

Figure 5: Evolution of temperature and HRR for a zero-dimensional case with isentropic compression. Initial conditions: pressure $=10.834 \mathrm{~atm}$, temperature $=677 \mathrm{~K}, \phi=0.3$. 
perature and HRR time evolution. Here, it is seen that the temperature starts to rise from the start even before any reaction occurs. This is the effect of isentropic compression. The initial pressure, temperature, and equivalence ratio for this case are $10.834 \mathrm{~atm}, 677 \mathrm{~K}$, and 0.3 . Note that although the initial temperature $\left(T_{o}=677\right.$ $\mathrm{K})$ is lower than the lower temperature case without isentropic compression $\left(T_{o}=\right.$ 743.5 K case in Fig. 4), the ignition behavior is very different and in fact resembles the combination of the low- and high-temperature cases. Fig. 6 shows the evolution of key species for this case. As presented in previous studies [9, 23], the key intermediate during the low-temperature first stage heat release is methoxymethylperoxy $\left(\mathrm{CH}_{3} \mathrm{OCH}_{2} \mathrm{O}_{2}\right)$. The second-stage or the intermediate-temperature regime corresponds to thermal dissociation of hydrogen peroxide $\left(\mathrm{H}_{2} \mathrm{O}_{2}\right)$. Finally, the third-stage corresponds to the typical high-temperature hydrogen ignition (via $\mathrm{H}$ $+\mathrm{O}_{2}$ branching reaction) [38].

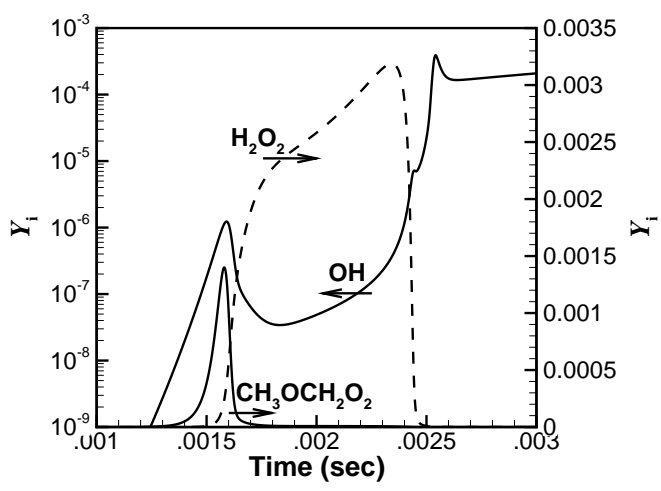

Figure 6: Evolution of key chemical species for a zero-dimensional case with isentropic compression. Initial conditions: pressure $=10.834$ atm, temperature $=677 \mathrm{~K}, \phi=0.3$. 


\section{Two-dimensional simulations}

\subsection{Effects of isentropic compression on scalar mixing}

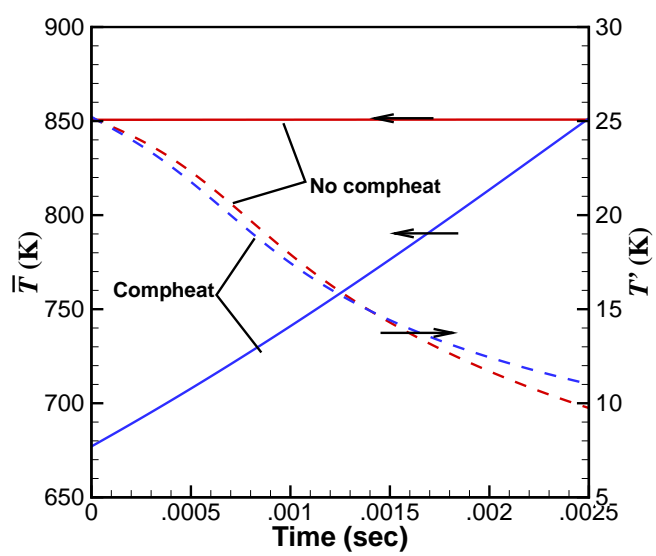

Figure 7: Evolution of mean and RMS of temperature for case a-NR-2D (Compheat) and b-NR-2D (No compheat).

Two-dimensional non-reacting mixing simulations were performed initially to assess the effects of isentropic compression (via inert mass source terms) on mixing of the temperature field, and in particular, its effect on the evolution of temperature gradients. For this purpose, a uniform initial composition with equivalence ratio 0.3 , and a random temperature field with hot and cold spots was initialized for cases with and without isentropic compression using the methodology described in Section 2. Isentropic compression affects the scalar and turbulence fields in the following ways. Firstly, due to the dependence of specific heat on local temperature, the hot and cold pockets experience differential increases in temperature due to isentropic compression. Secondly, the kinematic viscosity and hence the turbulence dissipation rate is a strong function of system pressure. Therefore, the mixing behavior is different with isentropic compression as the system pressure 


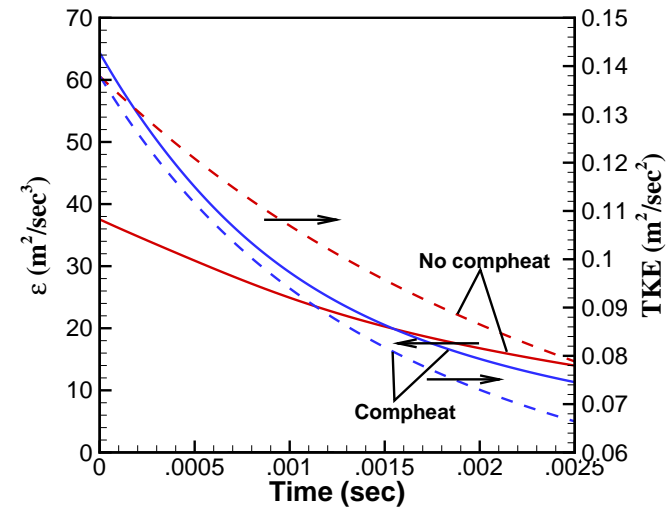

Figure 8: Evolution of turbulence dissipation rate $(\epsilon)$ and turbulence kinetic energy (TKE), for cases a-NR-2D (Compheat) and b-NR-2D (No compheat).

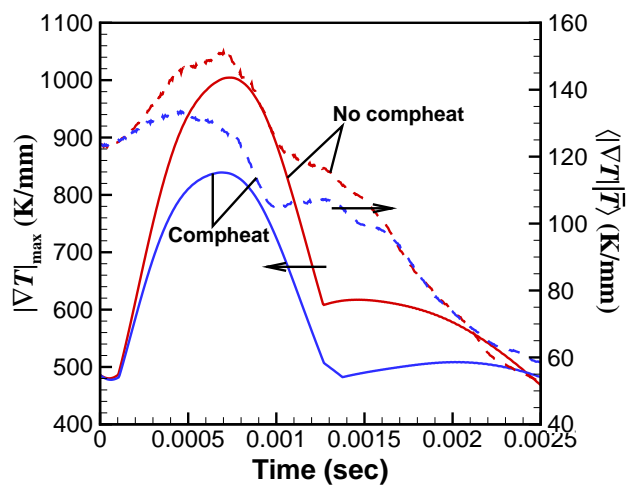

Figure 9: Evolution of maximum and conditionally averaged temperature gradient (conditional on mean temperature) in the domain, for cases a-NR-2D (Compheat) and b-NR-2D (No compheat). 
continually changes in time. To demonstrate and understand these effects, two cases, a-NR-2D and b-NR-2D, are simulated. For case a-NR-2D, the initial mean temperature and pressure are given in Table 1, and the initial turbulence and numerical parameters are given in Table 3. Fig. 7 shows the evolution of mean and RMS temperature fields for this case (this case is referred to as Compheat - illustrating the effect of isentropic compression heating). As expected, the mean temperature and pressure (not shown) increases with time due to isentropic compression, and the temperature RMS decays over time due to mixing. As will be shown later, the ignition delay for all cases studied is close to $2.5 \mathrm{msec}$. Therefore, to define an equivalent case without compression heating, the mean temperature and pressure at the end of $2.5 \mathrm{msec}(\bar{T}=851.43 \mathrm{~K}$, and $p=27.22 \mathrm{~atm})$ were chosen as initial conditions for case b-NR-2D (No compheat case). Fig. 7 also shows the $\bar{T}$ and $T^{\prime}$ evolution for this case. Here it is observed that, again as expected, $\bar{T}$ remains constant while $T^{\prime}$ decays over time. As the specific heat of cold pockets is smaller than that of hot pockets, the temperature rise is greater for cold pockets and less for hot pockets. This effect reduces $T^{\prime}$ as time progresses for case a-NR-2D. The somewhat faster decay of $T^{\prime}$ for the Compheat case at earlier times is partly attributed to this effect. Fig. 8 shows the temporal evolution of turbulence dissipation rate $(\epsilon)$ and turbulent kinetic energy (TKE) for the two cases. It is seen that higher viscosity (due to lower pressure) in the Compheat case results in a higher turbulence dissipation rate. This causes the TKE to decay faster for the Compheat case. These effects related to specific heat and viscosity cause the temperature gradients to evolve differently in the two cases. Fig. 9 shows the evolution of maximum temperature gradients in the domain and also the conditionally averaged temperature gradient (conditional on mean temperature) for the 
two cases. Note that both the maximum and conditionally averaged gradients are higher for the No compheat case compared to the Compheat case. While specific heat plays a minor role in reducing the temperature RMS, viscosity has a strong effect on the turbulence kinetic energy or $u^{\prime}$. Lower TKE due to higher turbulence dissipation rate results in a relatively reduced straining of the temperature field. Reduced turbulence straining in Compheat case is the principal reason behind the lower temperature gradients for this case compared to No compheat case. The magnitude of the temperature gradients have a major influence on the nature of the heat release mode [17], and therefore this discussion is important in understanding the combustion modes in HCCI engines in which isentropic compression heating is always present.

In the next section two-dimensional reacting simulations with isentropic compression heating are discussed.

\subsection{Effects of thermal and thermal-composition stratification}

In this section three cases with different initial stratification are considered: Case a-2D with only thermal inhomogeneities initially, case b-2D with both thermal and composition inhomogeneities with an uncorrelated $T-\phi$ field initially, and case c-2D also with both thermal and composition inhomogeneities with a negatively-correlated $T-\phi$ field initially. As mentioned in the Introduction, these different scenarios might exist in a practical engine depending on various parameters such as start of the injection timing, stratification in EGR, wall heat loss, and in-cylinder turbulence.

First, the mean (domain averaged) heat release rate for the three cases relative to the equivalent homogeneous case is examined. As given in Table 1, the initial mean $\phi$ for case a-2D is 0.3 , and for cases $b-2 D$ and c-2D, is 0.6 . Figure 10 
shows the mean heat release rate as a function of time for these three cases. It is observed that case a-2D generates heat release for both first and second stage ignition sooner than for the corresponding homogeneous case. The peak mean first stage heat release rate is lower for case a-2D compared to the homogeneous case. Moreover, it is noted that for case a-2D the mean heat release rate during the second and third stage ignition overlap in time, and therefore only a single peak is observed. It will be shown later that for this case the second and third stage ignition also overlap spatially such that at any instant both second and third stage ignition occurs at different locations in the domain. This is consistent with some of the findings in Ref. [23]. The peak third stage mean heat release rate is also lower than that for the corresponding homogeneous case. Case b-2D compared to the corresponding homogeneous case behaves in a similar fashion. Case c-2D, on the other hand, shows a different behavior. It more closely resembles the homogeneous case exhibiting a greater distinction between the second and third stage ignition peaks in the mean heat release rate. However, the first stage peak in the mean heat release rate is almost absent for this case. Also, the second and third stage ignition for this case occurs much earlier than the homogeneous case. Moreover, the peak third stage heat release rate is even higher than the homogeneous case. These observations can be explained by investigating the detailed spatial behavior of heat release presented next.

Figure 11 shows the heat release rate (HRR) field for the three cases: case a-2D (top row), case b-2D (middle row), and case c-2D (bottom row). Time increases from left to right: the figure on the left corresponds to the peak in the first stage heat release rate, the middle figure corresponds to a time when the integrated heat release is $50 \%$ of total heat release, and the figure on the right corresponds to an 
integrated heat release rate of $90 \%$ of the total heat release. It is observed that for all the three cases the first stage heat release occurs in thin reaction fronts. Note that at the $50 \%$ heat release point (middle column figures), for cases a-2D and b$2 \mathrm{D}$, heat release occurs in thin reaction fronts, whereas for case c-2D heat release occurs in thickened structures. Among cases a-2D and b-2D, case b-2D shows overall thinner reaction fronts than case a-2D. Differences in heat release rate structure are also seen at the $90 \%$ heat release point where cases a-2D and b-2D burn in thin reaction fronts whereas case c-2D burns more or less homogeneously. These findings are consistent with hydrogen/air results reported in Bansal and Im [21] where the uncorrelated $T-\phi$ case was also found to burn in the thin reaction front regime and the negatively correlated $T-\phi$ case was found to burn more homogeneously. It is interesting to note that these observations persist for a more complex DME fuel.

Next, the heat release rate (HRR) field is further investigated to identify the ignition modes present at the $50 \%$ and $90 \%$ heat release points. For this purpose, consider the results for case b-2D. Figure 12 shows the scatter plot of HRR vs. temperature at the $50 \%$ and $90 \%$ heat release points. This figure shows that at the $50 \%$ heat release point, HRR peaks at two distinct temperatures. At this time both intermediate- (second stage) and high-temperature (third stage) ignition occurs at different spatial locations. From these figures, HRR peaks at $1300 \mathrm{~K}$ and 1700 $\mathrm{K}$, for second stage and third stage ignition, respectively. The crossover temperature [38] between low and high temperature ignition chemistry for $\mathrm{H}_{2}$ oxidation was computed for the average pressure $(\bar{p}=60.843 \mathrm{~atm})$ at the time corresponding to the $50 \%$ heat release point. Equating the reaction rates for key branching and termination reactions [38] for $\mathrm{H}_{2}$ oxidation, the crossover temperature at this 
pressure was found to be $1421 \mathrm{~K}$, which lies between $1300 \mathrm{~K}$ and $1700 \mathrm{~K}$, further demonstrating that the second and third stage ignition mechanisms are controlled by hydrogen chemistry. The second stage corresponds to intermediate temperature $\mathrm{H}_{2} \mathrm{O}_{2}$ thermal dissociation and the third stage corresponds to high temperature branching through the reaction, $\mathrm{H}+\mathrm{O}_{2} \rightarrow \mathrm{OH}+\mathrm{H}$. Figure 12 shows that at a time corresponding to the $90 \%$ heat release point only the high temperature branch of heat release is occurring.

Next, the burning modes of the reaction fronts in the second and third stage are identified. The objective here is to distinguish between a steady deflagrative premixed flame and spontaneous ignition front propagation [30]. A steady premixed flame exhibits a balance between reaction and diffusion terms (in the species transport equations) in the reaction zone of the flame, whereas a spontaneous ignition front is dominated by reaction with negligible contribution from diffusion. The burning mode is determined by examining the magnitude of the reaction rate and diffusion terms in the $\mathrm{OH}$ mass fraction transport equation, traversing across a thin reaction front. $\mathrm{OH}$ is chosen because it is a key radical in hydrogen ignition chemistry which plays a dominant role in the second and third stage DME ignition. In Figure 13 the plot on the left shows the HRR field for case b-2D at the $50 \%$ heat release point. Colored isocontours are superimposed on the HRR field. These contours correspond to regions with temperature $\mathrm{T}=1300 \mathrm{~K}$ (green) and $1700 \mathrm{~K}$ (blue). Based on the present arguments, the green contour corresponds to the second stage ignition and the blue contour corresponds to the third stage ignition. It is interesting to note that the two different ignition chemistry pathways can exist in such close physical proximity to one another. Figure 13 also shows two cut plots, $\mathrm{a}$ and $\mathrm{b}$, traversing both ignition stages. In Figure 13, the plot for cut-a shows 
the reaction and diffusion terms in the $\mathrm{OH}$ transport equation together with HRR. The distance on the $\mathrm{x}$-axis goes from left to right (blue to green contour). The two peaks in the HRR cut plot correspond to the two reaction fronts. The cut plot shows that for the left HRR peak (at $1700 \mathrm{~K}$ ) the diffusion and reaction terms are of comparable magnitude, whereas for the right HRR peak (at $1300 \mathrm{~K}$ ) diffusion is negligible compared to reaction. This indicates that the left HRR peak (third stage ignition) is associated with burning in a premixed flame mode whereas the right HRR peak (second stage ignition) is associated with a spontaneous ignition front dominated by reaction alone.

In summary, there are two distinct differences between the two representative thin reaction fronts: first, the ignition chemistry that dominates the front is identified, that is, whether it corresponds to second or third stage DME ignition, and second, the mode of combustion is identified, that is, whether the fronts burn as a premixed deflagration wave or as a spontaneous ignition front. Since spontaneous ignition fronts travel at speeds much faster than premixed flames, the distance between the fronts changes rapidly. Moreover, it is also noted that the two interact in an upstream-upstream front interaction mode, such that the reactants of one front interact with the reactants of the other front. This is because, as seen in the cuta plot, the positive peaks of the reaction term (indicating reactants) for the two fronts are close to one another and the negative peaks (indicating products) are away from one another. The other cut plot, plot $b$, shows temperature and other key radicals again traversing through the two distinct reaction fronts. Here also, the distance along the $\mathrm{x}$-axis is from left to right in the HRR field plot, thus going from the $1700 \mathrm{~K}$ isocontour (corresponding to third stage ignition) to the 1300 $\mathrm{K}$ (corresponding to second stage ignition) isocontour. $\mathrm{H}_{2} \mathrm{O}_{2}$ has a much higher 
magnitude in the second stage ignition since it thermally dissociates into $\mathrm{OH}$ radicals during the second stage chemical runaway. $\mathrm{OH}$ on the other hand has a much higher magnitude coincident with the third-stage ignition peak because ignition here is dominated by high temperature branching reactions generating $\mathrm{OH}$. It is conceivable that owing to the close proximity of the two chemically distinct reaction fronts the radicals from one front are transported into the other and can alter the local chemistry.

Finally, in this section, the heat release rate history from the first to the third ignition stages is investigated by examining T- $\phi$ phase plots as a function of ignition progress. $\phi$ is defined based on the local mixture fraction [39] such that it is unaffected by reaction. Figure 14 shows scatter plots of T- $\phi$ colored by HRR for cases b-2D and c-2D. At early times (the left column plots), T- $\phi$ is still largely negatively correlated for case c-2D and remains uncorrelated for case b-2D. For both cases the peak first stage HRR occurs at a temperature of approximately 825 $\mathrm{K}$ and not at the highest temperature. This is a consequence of the dominant chemistry occurring during first stage ignition. For case b-2D, the peak HRR at this time occurs at the highest $\phi$ in the scatter, whereas, for case $c-2 D$, the peak HRR occurs at $\phi$ approximately 0.65 . For case c-2D the temperature at the richest mixture conditions is lower than it is at $\phi$ of 0.65 (since T- $\phi$ are negatively correlated for this case).

At later times, for both cases, T- $\phi$ correlations become increasingly correlated. This is a consequence of high $\phi$ regions having a higher calorific value and the temperature in high $\phi$ regions is higher after complete burnout. At the $50 \%$ heat release point (middle column) two distinct peaks are visible for both cases. The two peaks correspond to second and third stage ignition and occur at different 
temperatures as discussed earlier. At this time, high HRR occurs in fluid parcels with a high $\phi$ for a corresponding ignition temperature (approximately $1300 \mathrm{~K}$ for second stage and $1700 \mathrm{~K}$ for third stage ignition). At the $90 \%$ heat release point, only a single HRR peak occurs for both cases.

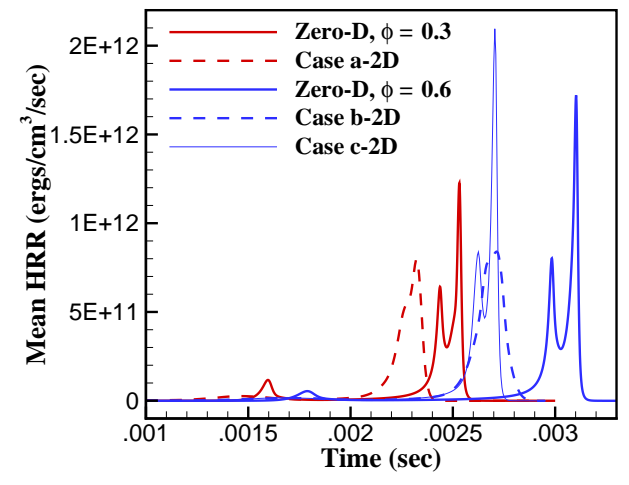

Figure 10: Mean heat release rate (HRR) for cases a-2D, b-2D, and c-2D, and two zero-D cases.

\subsection{Effects of turbulence}

In this section, the role turbulence plays in generating scalar gradients responsible for premixed flame propagation is ascertained. It will demonstrated that even if turbulence is frozen, as long as some scalar gradients exist prior to first stage ignition, then at later stages of ignition (during second and third stage) there are sufficient gradients in the radical fields generated solely due to reaction such that thin premixed flames are generated.

Figure 15 shows the HRR field for case d-2D (no turbulence, top row) and case e-2D (with turbulence, bottom row). Once again, time advances from left to right: left - time at peak first stage HRR, middle - time at $50 \%$ of total heat release, 


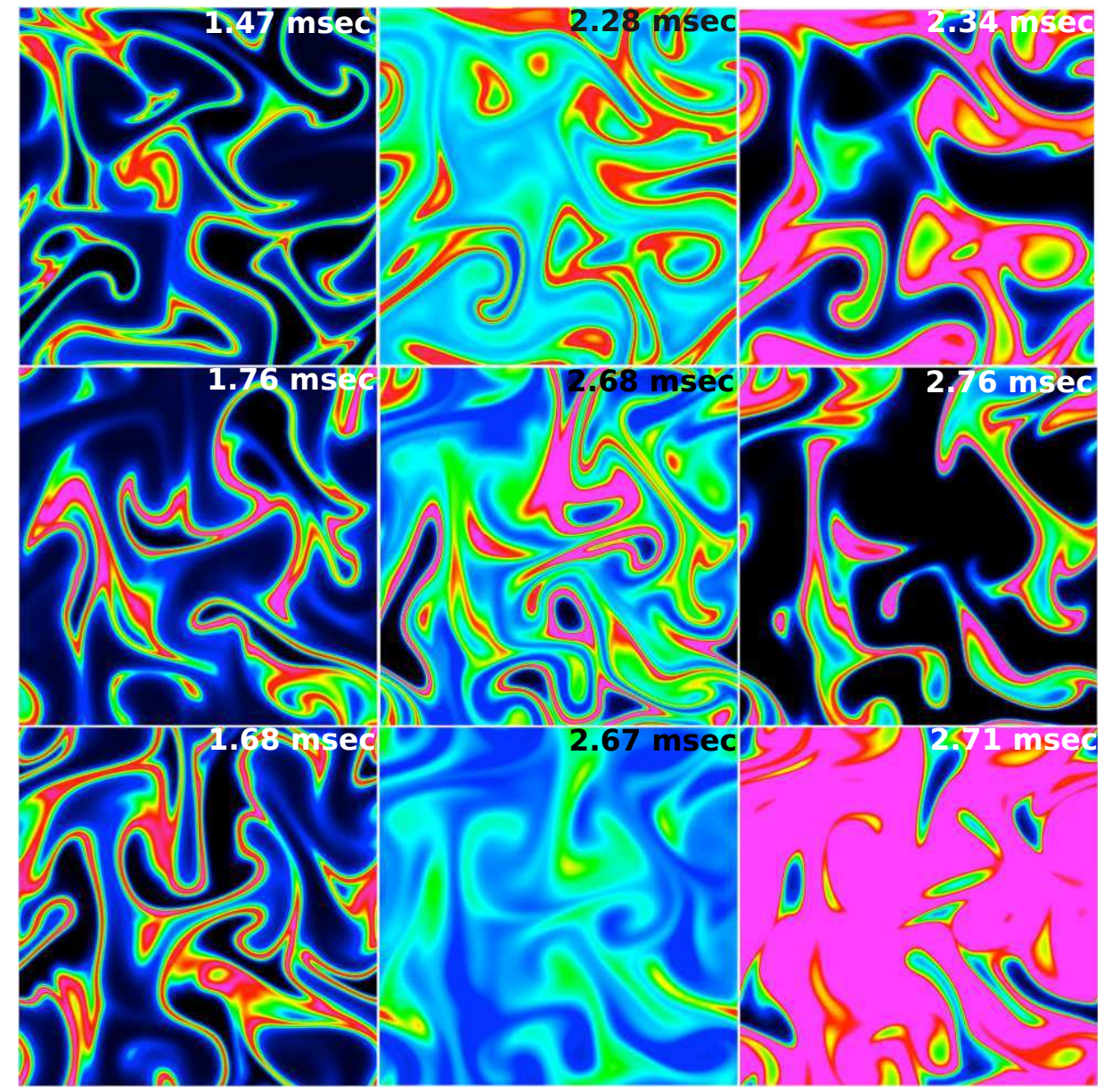

Figure 11: Heat release rate field (HRR) for cases a-2D, b-2D, and c-2D (top to bottom). The HRR magnitude varies between black (min value) and pink (max value). Time increases from left to right (times are shown in the plots): left - time corresponding to peak first stage heat release rate, middle and right - times corresponding to $50 \%$ and $90 \%$ of total heat release, respectively. 

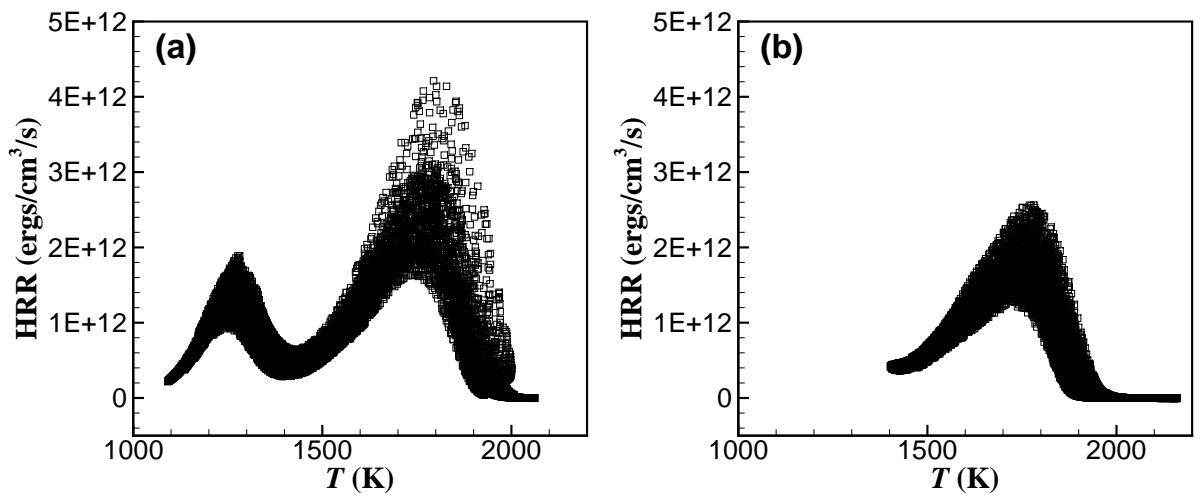

Figure 12: Scatter plot of HRR and temperature. (a) at a time corresponding to $50 \%$ of the total heat release, (b) at a time corresponding to $90 \%$ of the total heat release, for case b-2D.
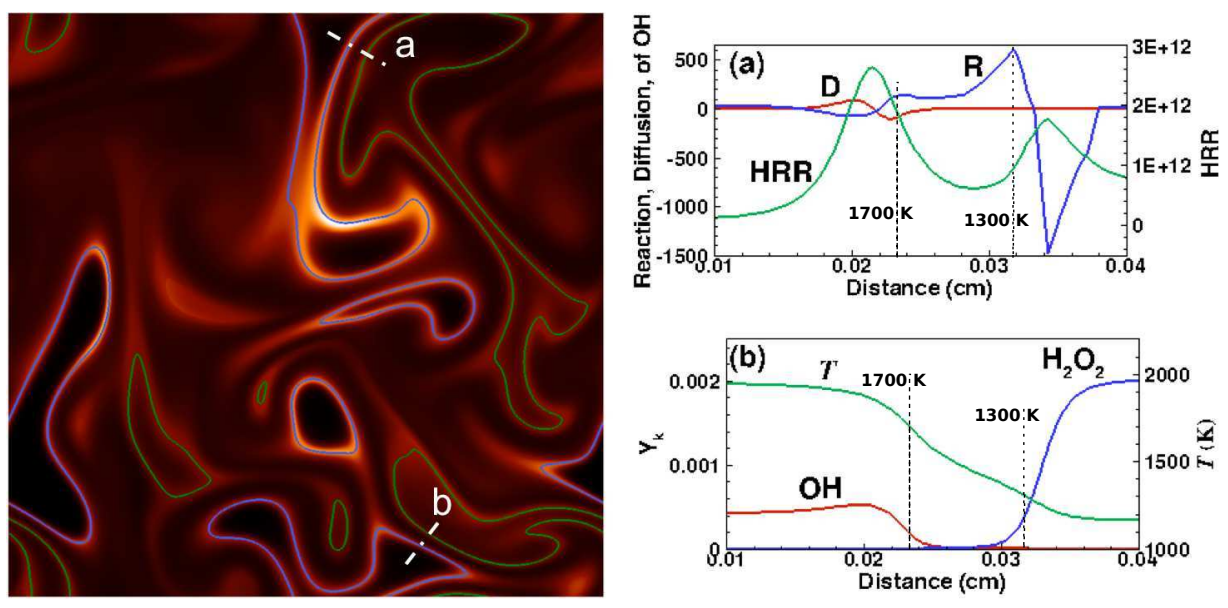

Figure 13: Left: Instantaneous HRR field (varies from black (min value) to white (max value)) at the $50 \%$ heat release point for case b-2D, with temperature isocontours overlaid: green $(T=1300$ $\mathrm{K})$, blue ( $T=1700 \mathrm{~K})$. Right: 1D cut plots as shown in the left plot. Cut a (top plot on right) shows reaction and diffusion terms in the $\mathrm{OH}$ species transport equation (both with units of $\frac{\mathrm{kg}}{\mathrm{m}^{3} \mathrm{~s}}$ ) and HRR $\left(\frac{\mathrm{ergs}}{\mathrm{cm}^{3} \mathrm{~s}}\right)$ as a function of distance along the cut (left to right), cut b (bottom plot on right) shows key species mass fraction and temperature as a function of distance (left to right). Vertical dashed lines in the cut plots indicate spatial locations of $T=1300 \mathrm{~K}$ and $1700 \mathrm{~K}$. 

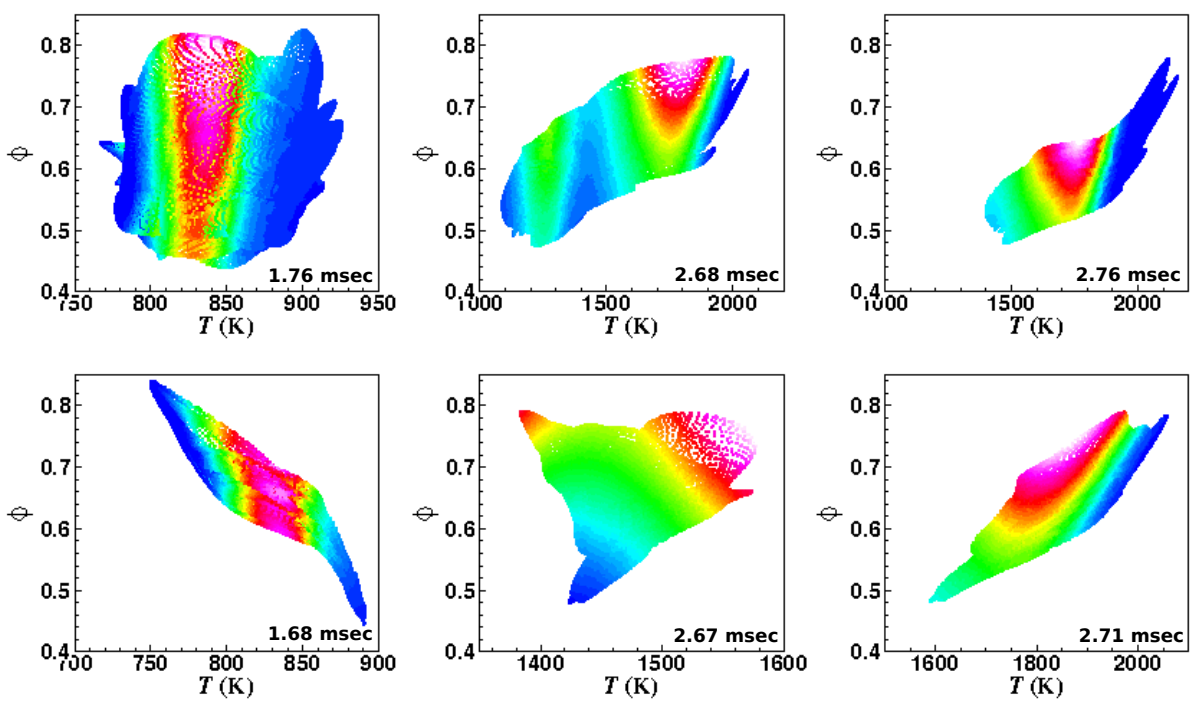

Figure 14: Scatter plots of $\phi$ with temperature, colored by HRR (colormap varies from blue (lowest HRR) to green to red to pink to white (highest HRR)). The top row corresponds to case b-2D, and the bottom row corresponds to case c-2D. Time increases from left to right and are the same as those in Fig. 11, i.e. time increases from left to right going from the time corresponding to peak first stage HRR, $50 \%$ of total integrated heat release, and $90 \%$ of total integrated heat release. 
and right - time at $90 \%$ of total heat release. In case d-2D there are no velocity fluctuations initially and hence the flow is quiescent initially. For both cases we observe that during the first stage of ignition thin fronts exist. Even at later stages of ignition we note that case d-2D (with no turbulence) shows the presence of thin reaction fronts similar to that for case e-2D. Since there is no turbulence in case $d-2 D$, this shows that the reaction generated gradients in the scalar fields are strong enough to lead to the formation of thin reaction fronts. This is interesting for two reasons: First, from a practical stand point this suggests that in real engines there is a likelihood that reaction generated gradients dominate the heat release process and therefore premixed flames may exist in real engines even if turbulence is weak. Recall that the mixing time scale in the present DNS is comparable to that in a real engine, although the integral length scale and turbulence intensities are proportionately smaller by a factor of about five. Hence, the ratio of mixing to ignition delay time scales is preserved. Secondly, from a RANS/LES modelling standpoint, this implies that the turbulence dissipation rate and reactive scalar dissipation rate may not be strongly correlated. Moreover, the passive scalar dissipation rate may be uncorrelated with reactive scalar dissipation rate. These considerations must be kept in mind when developing models for scalar dissipation rates of scalars in compression ignition combustion environments. Figure 16 further shows the integrated heat release for the two cases and also for an equivalent homogeneous case. Although the peak heat release rate is higher for case d-2D compared to case e-2D, these cases are still qualitatively comparable, as both cases exhibit an overlap between the second and third ignition stages. The figure further demonstrates that even without turbulence case d-2D does not burn homogeneously. 


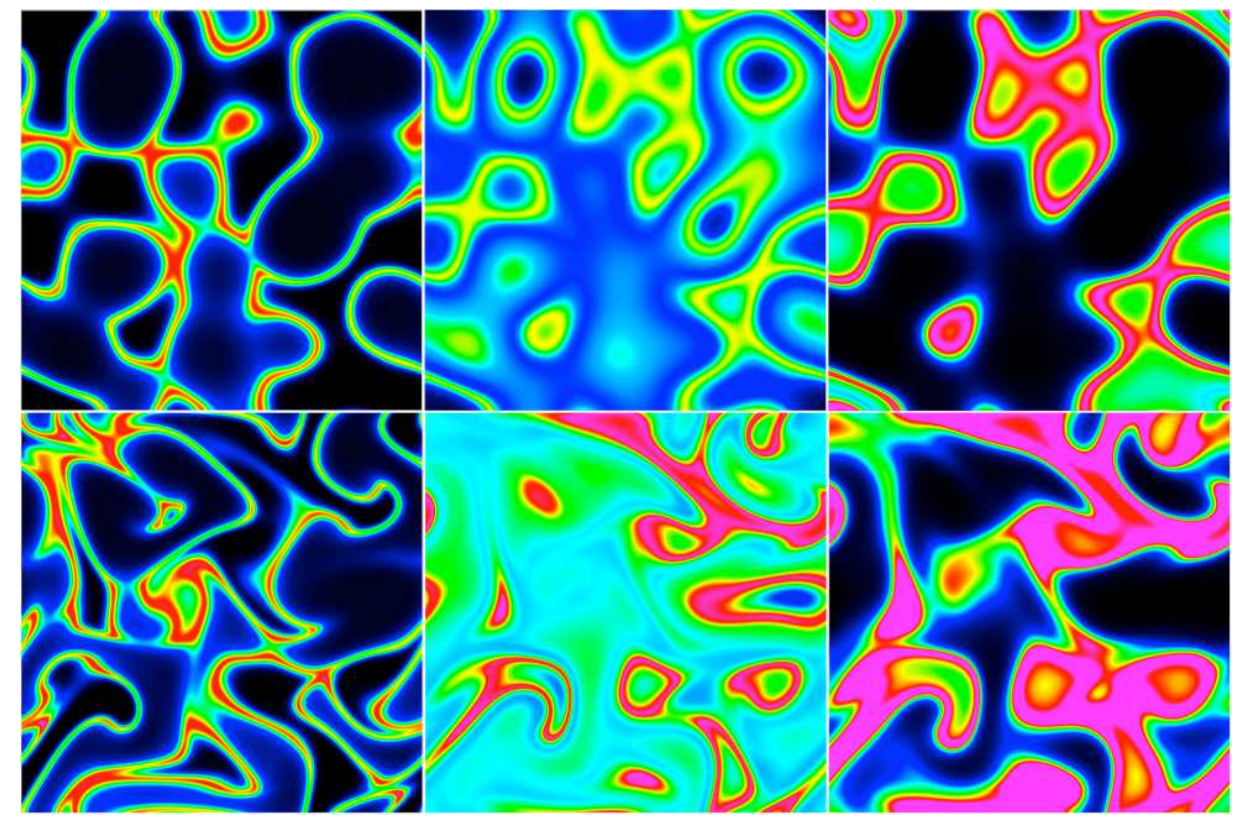

Figure 15: HRR field for cases d-2D (top row) and e-2D (bottom row). HRR magnitude varies from black (min value) to pink (max value). Time increases from left to right: left - time corresponding to peak first stage heat release rate, middle - time at $50 \%$ heat release point, and right time at $90 \%$ heat release point.

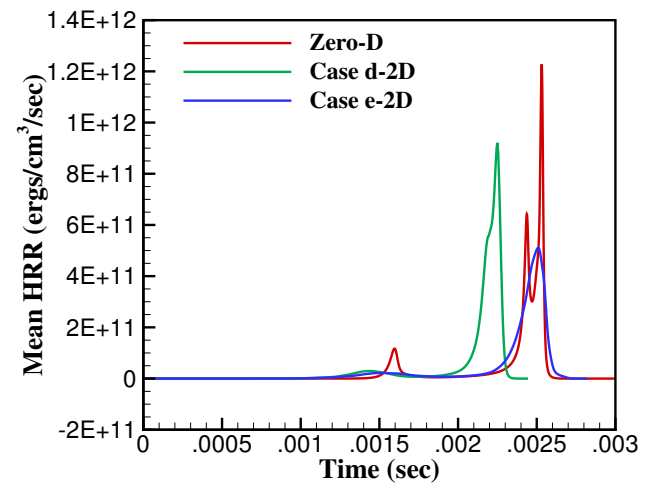

Figure 16: Mean HRR history for cases d-2D and e-2D and for a zero-dimensional case. 


\section{Three-dimensional simulation}

In this section, the results of the three-dimensional simulation are presented. The initial conditions and the numerical setup are defined in Section 2. 3-D turbulence is inherently different from 2-D turbulence because of the presence of vortex-stretching term in the 3-D case. This results in a wider range of scales and greater wrinkling of fronts for the 3-D case. In this section we will do an indepth investigation of the heat release modes, dominant chemistry, and dissipation structures during the second- and third-stage DME autoignition for a 3-D case.

Fig. 17 shows a scatter plot of $\mathrm{CH}_{3}$ mass fraction and temperature at time, $t=2.15 \mathrm{~ms}$ for the $3-\mathrm{D}$ case. Note the existence of two distinct peaks in $\mathrm{CH}_{3}$ mass fraction at this time. They correspond to the intermediate (2nd stage) and high (3rd stage) ignition chemistry, as was also discussed for the 2-D simulation earlier. Figs. 18 and 19 show the colormap of temperature and $\mathrm{CH}_{3}$ mass fraction at this time. In both figures the isosurface contour at high values of $\mathrm{CH}_{3}$ is plotted. Temperature isosurface contours corresponding to $1300 \mathrm{~K}$ and $1500 \mathrm{~K}$ are plotted in Figs. 18 and 19, respectively. At both these temperature values, a local peak in $\mathrm{CH}_{3}$ mass fraction is observed.

Fig. 20 next shows the diffusion and reaction rate terms in the $\mathrm{OH}$ mass fraction conservation equation. The pink surface is the region of high $\mathrm{OH}$ dissipation rate. We note that regions of high dissipation rate occur in the form of thin "platelets" (also referred to as "pancake-like" later in the paper). These high dissipation regions are found to be engulfed in the green color isosurface contour

of the reaction rate. It is interesting to note that the magnitude of reaction and diffusion in these high dissipation regions are approximately comparable (both with a value of approximately 20). Figs. 21 and 21 show two zoomed in views 
of such high dissipation regions. These figures indicate that the regions of high dissipation are burning in a premixed deflagrative mode with equal contribution from diffusion and reaction instead of being dominated solely by reaction. The regions away from these high dissipation structures are burning in a chemically dominant mode corresponding to spontaneous ignition.

The nature of these premixed deflagation modes is further clarified next. Fig. 23 shows the same high dissipation rate regions together with the temperature field. Two isosurface contours for temperature are plotted: $1300 \mathrm{~K}$ and $1500 \mathrm{~K}$. This figure shows that regions of high dissipation rate are engulfed in the regions where the temperature is $1500 \mathrm{~K}$. Zoomed in figures, Figs. 24 and 25 further demonstrate this. Together, Figs. 20 and 23 suggest that premixed deflagrative combustion mode is present in regions of high temperature, which in turn is dominated by high temperature ignition chemistry. These findings are consistent with the results from the 2-D simulation discussed in earlier sections.

Fig. 26 shows the scatter plot of $\mathrm{CH}_{3}$ mass fraction and temperature at a later time of $2.175 \mathrm{~ms}$. At this time, the second stage or intermediate temperature ignition mode starts to disappear and the majority of the domain is burning in the third stage or high temperature ignition mode. Fig. 27 shows the diffusion and reaction rate terms at this time in a plot similar to Fig. 20. Note that a few additional high dissipation "pancake-like" regions are formed. This is an indication that reaction generated gradients are the source of the formation of these high dissipation regions. Turbulence is not causing these high dissipation regions since turbulence is decaying with time, whereas these high dissipation regions increase in frequency with time. Figs. 28 and 29 show some zoomed in high dissipation rate regions. Once again note that close to these high dissipation regions, the magnitudes of 
reaction and diffusion are comparable.

Finally, Fig. 30 is a scatter plot of $\mathrm{CH}_{3}$ and temperature at an even later time of $2.20 \mathrm{~ms}$. At this time, only one peak in $\mathrm{CH}_{3}$ mass fraction exists, occurring at 1500 K. This corresponds to high temperature ignition chemistry. Fig. 31 demonstrates this further in a colormap field plot. At this time the high $\mathrm{CH}_{3}$ regions occur in more concentrated discrete regions compared to that at $t=2.15 \mathrm{~ms}$. Fig. 32 shows diffusion and RR field plots at this time. Many more high dissipation rate regions emerge by this time. Figs. 33 and 34 show the zoomed in view of high dissipation regions. The high dissipation regions are always present next to a reaction rate region of comparable magnitude.

In summary, we have observed in these 3-D simulations that during the later times two distinct ignition stages emerge. These correspond to intermediate and high temperature DME ignition chemistry. As the ignition progresses from second to third stage, more and more regions of high dissipation in the form of thin pancakes emerge. These are formed due to reaction generated gradients rather than turbulence itself and are always in the vicinity of regions where the reaction rate magnitude is comparable to the diffusion term magnitude inside of these pancakes. Thus, the regions inside and near to these high dissipation regions are burning in a premixed deflagrative mode rather than in a chemistry dominated spontaneous ignition mode. 


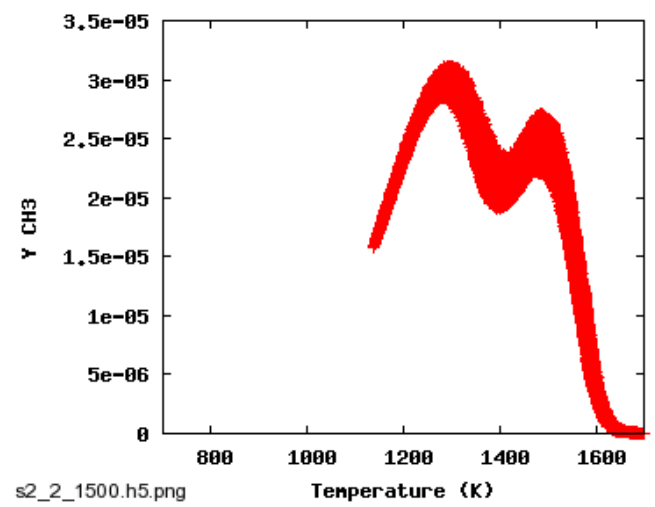

Figure 17: Scatter plot of $Y_{\mathrm{CH} 3}$ and temperature (K) at $t=2.15 \mathrm{~ms}$.

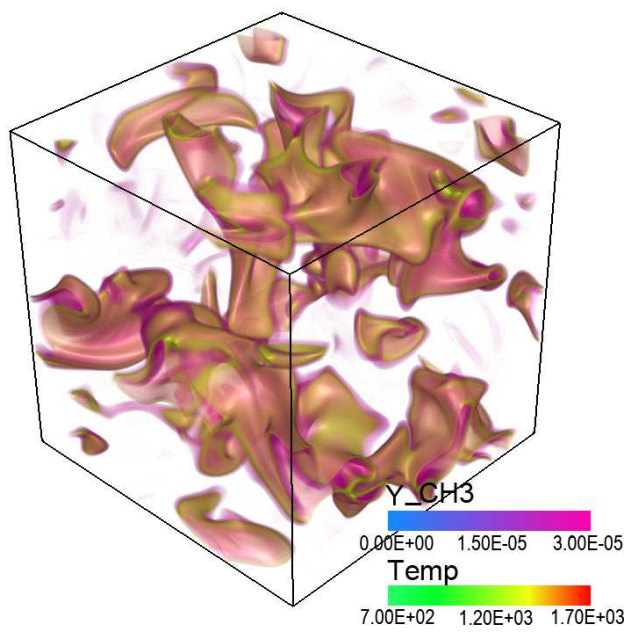

Figure 18: Colormap image of $Y_{\mathrm{CH} 3}$ and temperature (K) at $t=2.15 \mathrm{~ms}$. Iso-surface contours are shown for temperature of $1300 \mathrm{~K}$ and peak $Y_{\mathrm{CH} 3}$. 


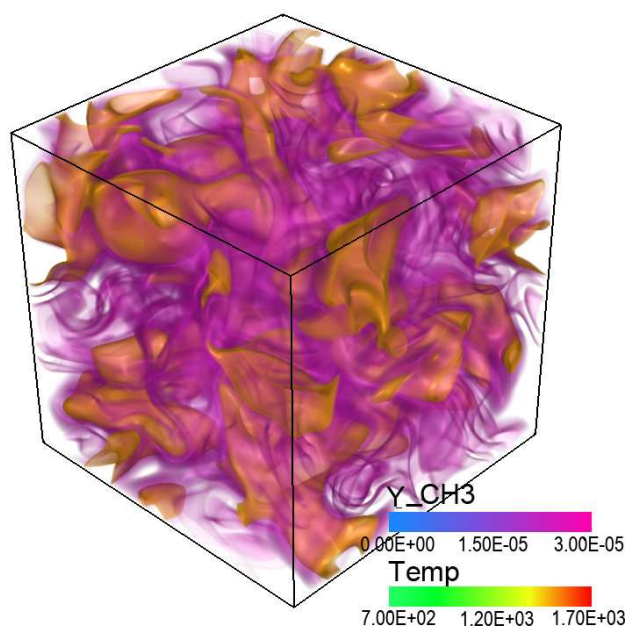

Figure 19: Colormap image of $Y_{\mathrm{CH} 3}$ and temperature $(\mathrm{K})$ at $t=2.15 \mathrm{~ms}$. Iso-surface contours are shown for temperature of $1500 \mathrm{~K}$ and peak $Y_{\mathrm{CH} 3}$.

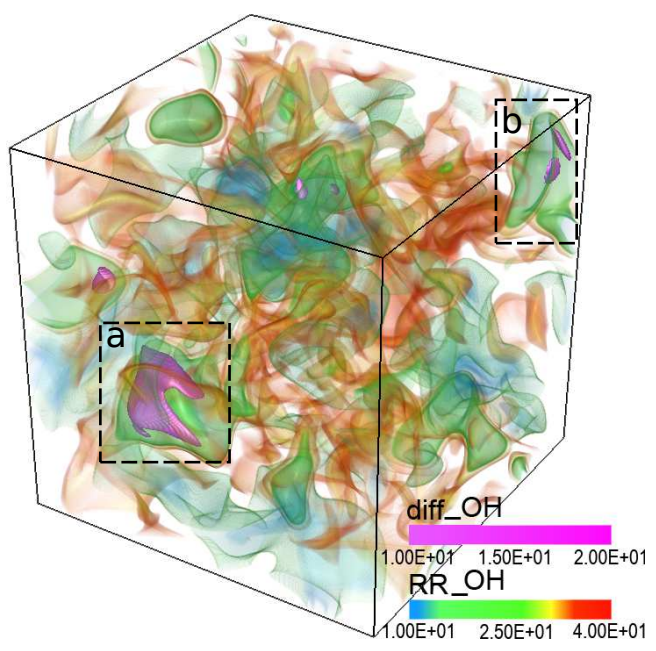

Figure 20: Colormap image of diffusion and reaction rate terms (both have units of $\frac{\mathrm{kg}}{\mathrm{m}^{3} \mathrm{~s}}$ ) in the $\mathrm{OH}$ mass fraction conservation equation at $t=2.15 \mathrm{~ms}$. 


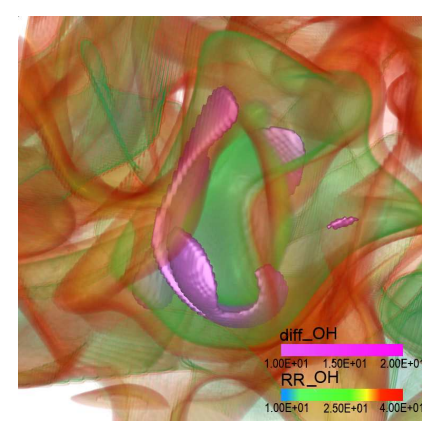

Figure 21: Zoomed in figure, corresponding to the inset a of Fig. 20.

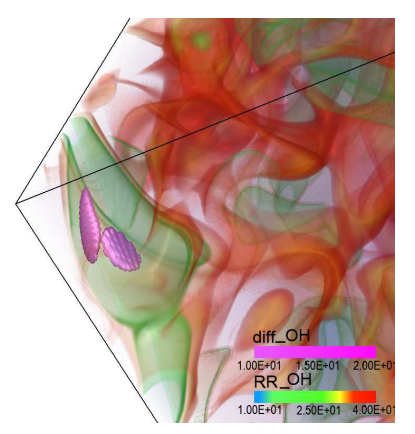

Figure 22: Zoomed in figure, corresponding to the inset b of Fig. 20. 


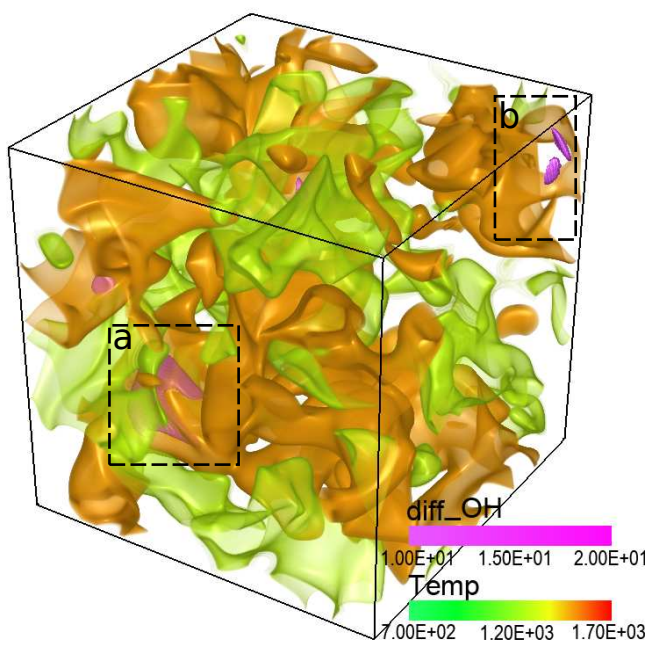

Figure 23: Colormap image of the diffusion term $\left(\frac{\mathrm{kg}}{\mathrm{m}^{3} \mathrm{~s}}\right)$ in the $\mathrm{OH}$ mass fraction conservation equation and temperature $(\mathrm{K})$ at $t=2.15 \mathrm{~ms}$. Two temperature iso-surface contours are shown at $1300 \mathrm{~K}$ and $1500 \mathrm{~K}$.

\section{Conclusions}

In this study we performed direct numerical simulations of autoignition of dimethyl ether (DME) in high pressure stratified turbulent mixtures. Both thermal and concentration stratification were considered. A reduced chemical mechanism consisting of 30 species was used. Inert mass source terms were added to the governing equations to mimic isentropic pressure rise that occurs in a practical internal combustion engine. A variety of two-dimensional simulations were conducted first by varying key temperature and composition parameters, and subsequently a three-dimensional simulation was also performed.

DME autoignition was found to be a complex three-staged phenomena, each stage corresponding to a distinct chemical pathway. The first stage corresponds to fuel-specific low temperature ignition where the key intermediate radical is 


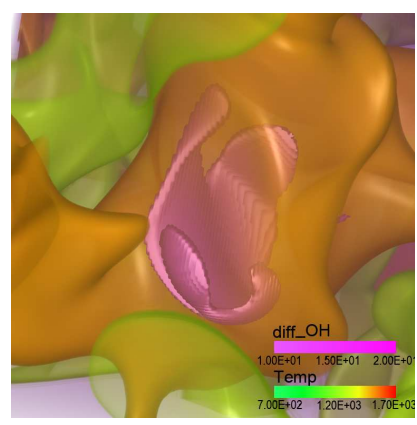

Figure 24: Zoomed in figure, corresponding to the inset a of Fig. 23.

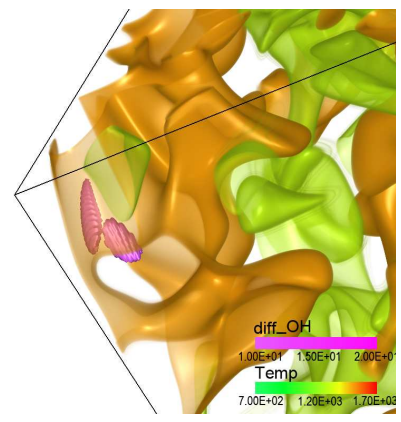

Figure 25: Zoomed in figure, corresponding to the inset b of Fig. 23.

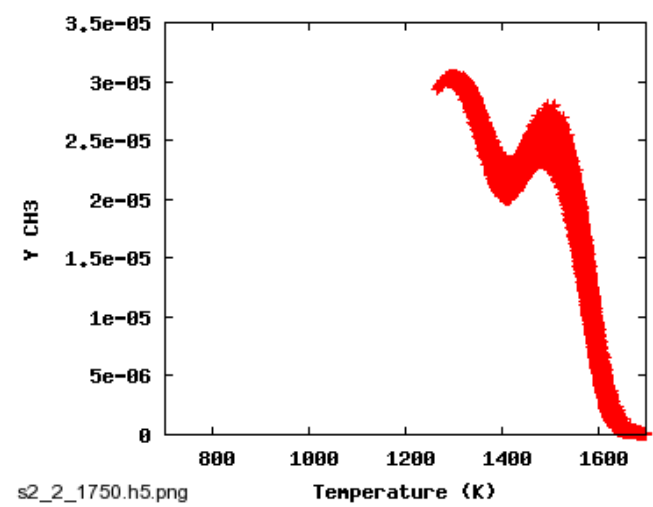

Figure 26: Scatter plot of $Y_{\mathrm{CH} 3}$ and temperature (K) at $t=2.175 \mathrm{~ms}$. 


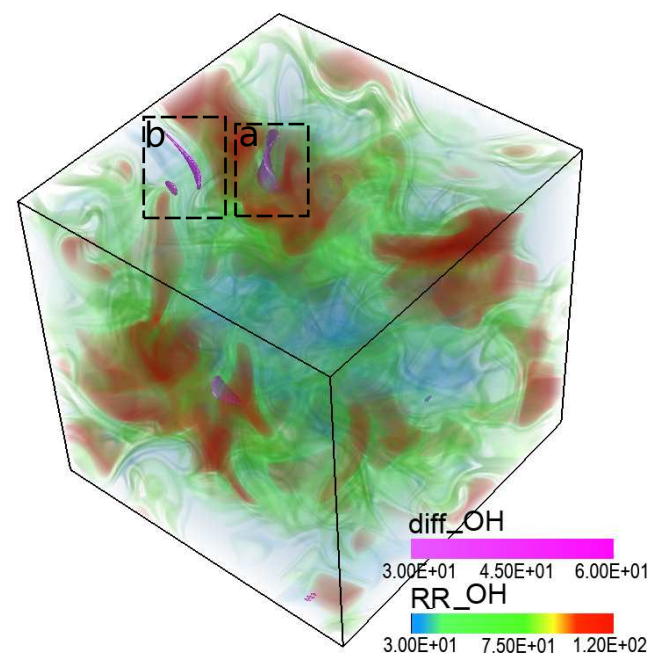

Figure 27: Colormap image of diffusion and reaction rate terms (both have units of $\frac{\mathrm{kg}}{\mathrm{m}^{3} \mathrm{~s}}$ ) in the $\mathrm{OH}$ mass fraction conservation equation at $t=2.175 \mathrm{~ms}$.

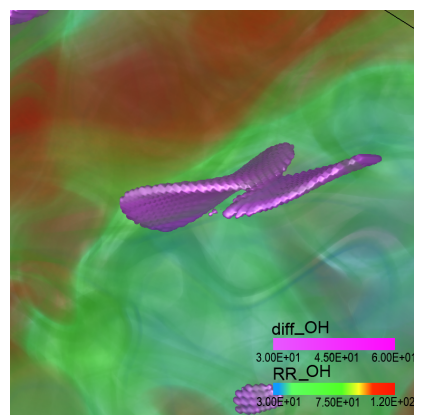

Figure 28: Zoomed in figure, corresponding to the inset a of Fig. 27. 


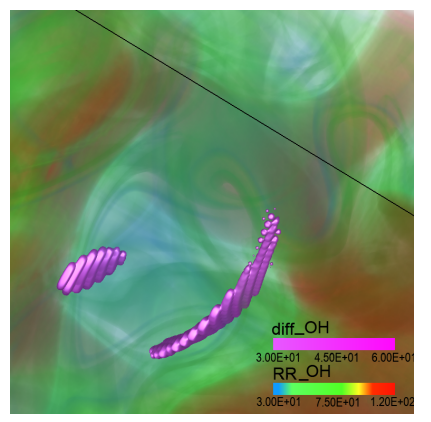

Figure 29: Zoomed in figure, corresponding to the inset b of Fig. 27.

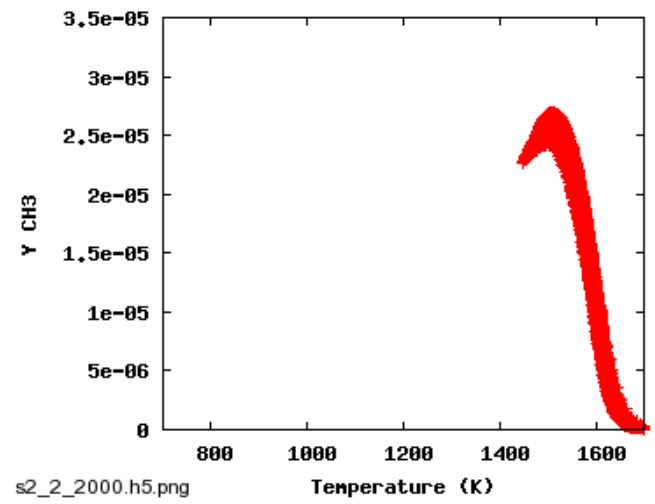

Figure 30: Scatter plot of $Y_{\mathrm{CH} 3}$ and temperature at $t=2.20 \mathrm{~ms}$. 


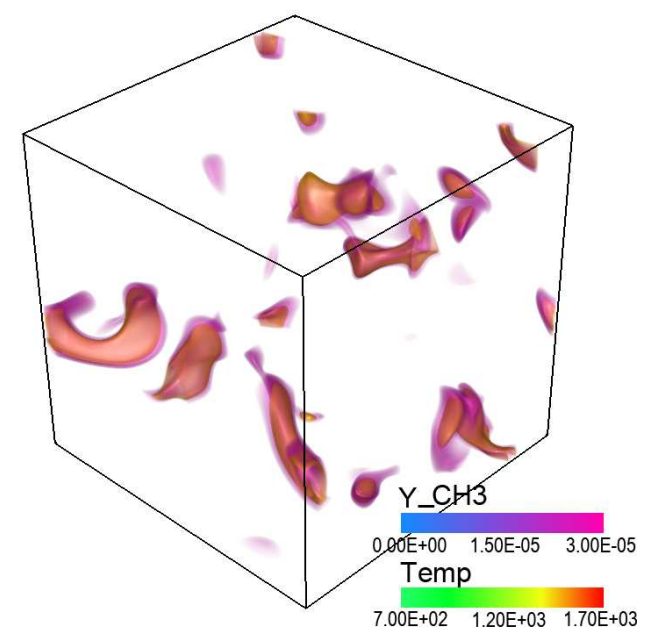

Figure 31: Colormap image of $Y_{\mathrm{CH} 3}$ and temperature at $t=2.20 \mathrm{~ms}$. Iso-surface contours shown for temperature of $1500 \mathrm{~K}$ and peak $Y_{\mathrm{CH} 3}$.

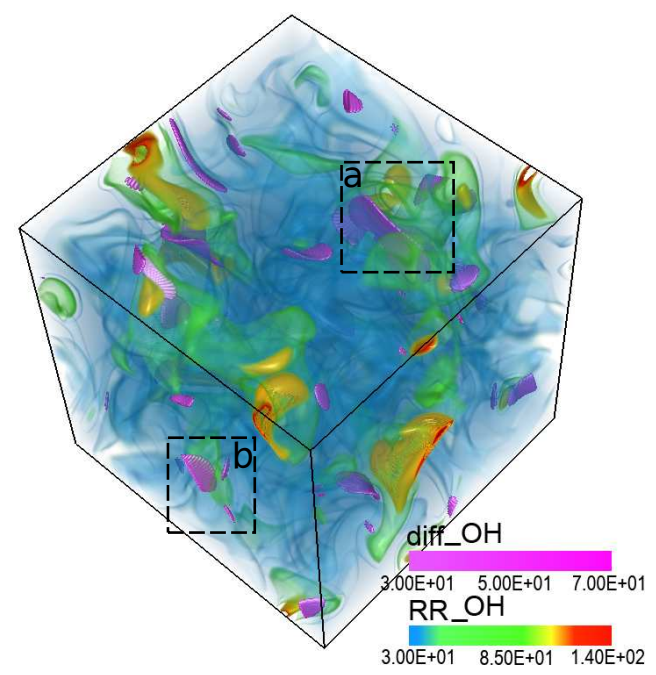

Figure 32: Colormap image of the diffusion and reaction rate terms (both have units of $\frac{\mathrm{kg}}{\mathrm{m}^{3} \mathrm{~s}}$ ) in the $\mathrm{OH}$ mass fraction conservation equation at $t=2.20 \mathrm{~ms}$. 


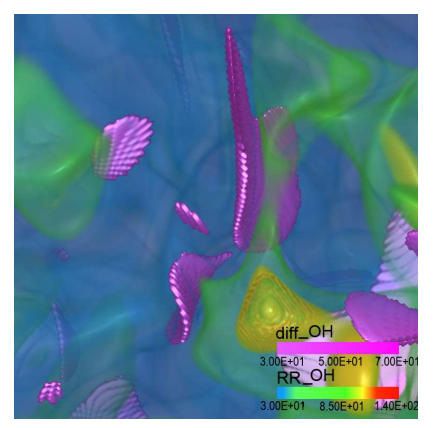

Figure 33: Zoomed in figure, corresponding to the inset a of Fig. 32.

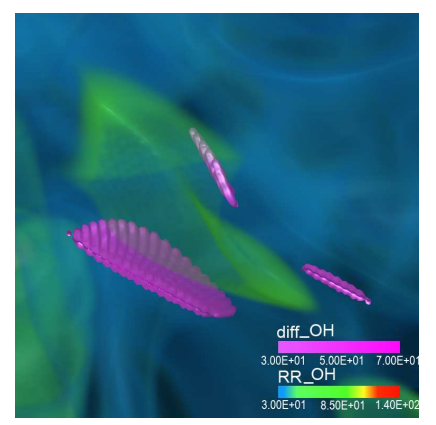

Figure 34: Zoomed in figure, corresponding to the inset b of Fig. 32. 
$Y_{\mathrm{CH} 3 \mathrm{OCH} 2 \mathrm{O} 2}$. The second and third stage ignition pathways correspond predominantly to intermediate and high temperature hydrogen ignition: the second stage is dominated by $\mathrm{H}_{2} \mathrm{O}_{2}$ dissociation, whereas the third stage is governed by the classical chain branching-termination balance.

First, a 2-D parametric study investigated the effects of isentropic compression on mixing statistics in a non-reacting turbulent stratified mixture. The compression heating case is found to have lower temperature gradients compared to the case without compression. Various factors are discussed in this regard including the role of specific heat in altering the temperature RMS and the fluid viscosity changes due to pressure rise which, in turn, alter the turbulence field.

Next, a reacting 2-D parametric study is conducted to investigate the different kinds of stratification. Three cases are simulated: a thermal stratified case, an initially uncorrelated temperature-equivalence ratio stratified case, and lastly, an initially negatively correlated temperature-equivalence ratio stratified case. It is found that the uncorrelated case leads to formation of thinner reaction fronts which behave like conventional premixed deflagrations, whereas the negativelycorrelated case leads to predominantly homogeneous ignition. For the uncorrelated case, it was found that the second and third ignition stages occur in close proximity in space and time to one another, and therefore, there can be chemical and diffusive interaction between the two stages. It was also found that large gradients in radical concentrations are generated due to reaction during the third stage. This causes a transition in the nature of the front from a spontaneously igniting chemistry driven front in the second stage to a premixed deflagrative front in the third stage.

Next, we investigated the direct effects of turbulence on autoignition phenom- 
ena. For this we conducted a simulation with frozen turbulence in which the velocity fluctuations were absent initially. However, even in the absence of turbulence, scalar gradients were generated due to reaction during the later stages of ignition increasing the role of diffusion and leading to the formation of thin reaction fronts. This indicates that thin flame like structures can still form in autoigniting mixtures in practical engine environments even if the underlying turbulence is weak.

Finally, results were presented for a 3-D thermally stratified simulation which provided detailed descriptions of the geometrical structure of high dissipation rate regions and reaction regions around them. Overall, the conclusions from the 3-D simulation were found to be qualitatively similar to those from 2-D simulations.

\section{Acknowledgments}

The research at Sandia National Laboratories is supported by the Combustion Energy Frontier Research Center, an Energy Frontier Research Center funded by the US Department of Energy (DOE), Office of Science, Office of Basic Energy Sciences under Award No. DE-SC0001198. Computer allocations were awarded by DOEs Innovative and Novel Computational Impact on Theory and Experiments (INCITE) Program. Sandia National Laboratories is a multiprogram laboratory operated by Sandia Corporation, a Lockheed Martin Company, for the US Department of Energy under contract DE-AC04-94-AL85000. This research used resources of the National Center for Computational Sciences at Oak Ridge National Laboratory (NCCS/ORNL) which is supported by the Office of Science of the US DOE under Contract No. DE-AC05-00OR22725. We thank Prof. Hongfeng Yu of University of Nebraska-Lincoln for generating some of the visualizations for the 3-D simulations. 


\section{Appendix A}

The derivation of inert mass (density) source terms which mimic the pressure rise due to isentropic compression resulting from piston motion in realistic engines is given in this Appendix. Considering only the isentropic compression effect, the local energy equation simplifies to:

$$
\rho c_{p} \frac{d T}{d t}=\frac{d P}{d t}
$$

Note that in deriving the inert mass source term we are neglecting the effects of any convective, diffusive, or reaction terms and thus Eq. 2 does not have these terms. The equation of state is used to obtain the rate of change of pressure:

$$
\begin{aligned}
P & =\rho R T \\
\frac{d P}{d t} & =\rho R \frac{d T}{d t}+R T \frac{d \rho}{d t}
\end{aligned}
$$

Substituting for $\frac{d T}{d t}$ from Eq. 3 b in Eq. 2:

$$
\begin{aligned}
& \frac{d \rho}{d t}=\frac{1}{R T} \frac{d P}{d t}\left(1-\frac{R}{c_{p}}\right) \\
& \frac{d \rho}{d t}=\frac{1}{\gamma R T} \frac{d P}{d t} \\
& \frac{d \rho}{d t}=\frac{1}{c^{2}} \frac{d P}{d t}
\end{aligned}
$$

Therefore, the local density source term is inversely proportional to the square of local sound speed. Integrating Eq. 4 over the entire DNS volume, and dividing by total volume $V$ ( $V$ is constant in DNS):

$$
\begin{aligned}
\frac{d}{d t}\left(\frac{1}{V} \int \rho d V\right) & =\frac{d P}{d t}\left(\frac{1}{V} \int \frac{1}{c^{2}} d V\right) \\
\frac{d \bar{\rho}}{d t} & =\frac{d P}{d t} \overline{\left(\frac{1}{c^{2}}\right)}
\end{aligned}
$$




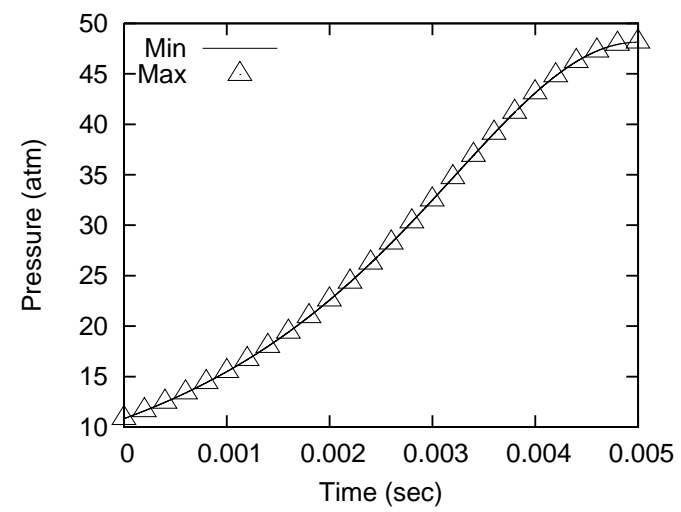

Figure 35: Min and max pressure evolution in the domain for a thermally inhomogeneous nonreacting $2 \mathrm{D}$ case due to isentropic compression heating.

Dividing Eq. 4 by Eq. 5, we get an equation for the local density source term:

$$
\frac{d \rho}{d t}=\frac{1 / c^{2}}{\overline{1 / c^{2}}} \frac{d \bar{\rho}}{d t}
$$

where, $\frac{d \bar{\rho}}{d t}$, the global rate of change of density, is given by the crank-slider relation for engines [40].

To demonstrate the effect of these source terms on bulk pressure evolution, Fig. 35 shows the evolution of minimum and maximum pressure within the domain for a thermally inhomogeneous non-reacting $2 \mathrm{D}$ case when these source terms are included. Note that the bulk pressure variation within the domain at any particular instant is almost negligible (min and max pressure plots lie on top of each other) when compared to the entire dynamic range of the pressure evolution in time.

\section{References}

[1] F. Zhao, T. Asmus, D. Assanis, J. Dec, J. Eng, P. Najt, Homogeneous Charge Compression Ignition (HCCI) Engines, Key Research and Development Is- 
sues, Society of Automotive Engineers, Inc., Warrendale, Pennsylvania, 2002.

[2] K. Epping, S. Aceves, R. Bechtold, J. Dec, SAE Technical Paper 2002-011923 (2002).

[3] M. Sjöberg, J. E. Dec, N. P. Cernansky, SAE Technical Paper 2005-01-0113 (2005).

[4] A. Babajimopoulos, G. A. Lavoie, D. Assanis, SAE Technical Paper 200301-3220 (2003).

[5] R. Hasegawa, H. Yanagihara, SAE Technical Paper 2003-01-0745 (2003).

[6] M. Sjöberg, J. E. Dec, SAE Intl. J. Fuels Lubricants 5 (2012) 7-27.

[7] K. Komatsu, M. Asanuma, A. Lijima, K. Yoshida, H. Shoji, SAE Intl. J. Engines 5 (2012) 9-16.

[8] S. H. Park, C. S. Lee, Prog. Energy Combust. Sci. 39 (2013) 147-168.

[9] H. Yamada, K. Suzaki, H. Sakanashi, N. Choi, A. Tezaki, Combust. Flame 140 (2005) 24-33.

[10] H. J. Curran, S. L. Fischer, F. L. Dryer, Int. J. Chem. Kinetics 32 (2000) $741-759$.

[11] H. Yamada, K. Suzaki, A. Tezaki, Y. Goto, Combust. Flame 154 (2008) $248-258$.

[12] Z. Zhao, M. Chaos, A. Kazakov, F. L. Dryer, Int. J. Chem. Kinetics 40 (2008) $1-18$. 
[13] C. Funk, V. Sick, D. L. Reuss, W. J. A. Dahm, SAE Technical Paper 200201-2841 (2002).

[14] J. E. Dec, W. Hwang, SAE Technical Paper 2009-01-0650 (2009).

[15] S. Sreedhara, K. N. Lakshmisha, Proc. Combust. Inst. 29 (2002) 2051-2059.

[16] T. Echekki, J. H. Chen, Combust. Flame 134 (2003) 169-191.

[17] R. Sankaran, H. G. Im, E. R. Hawkes, J. H. Chen, Proc. Combust. Inst. 30 (2005) 875-882.

[18] J. H. Chen, E. R. Hawkes, R. Sankaran, S. D. Mason, H. G. Im, Combust. Flame 145 (2006) 128-144.

[19] E. R. Hawkes, R. Sankaran, P. P. Pébay, J. H. Chen, Combust. Flame 145 (2006) 145-159.

[20] C. S. Yoo, T. Lu, J. H. Chen, C. K. Law, Combust. Flame 158 (2011) $1727-$ 1741.

[21] G. Bansal, H. G. Im, Combust. Flame 158 (2011) 2105-2112.

[22] H. A. El-Asrag, Y. Ju, Combust. Theory Modelling 17 (2013) 316-334.

[23] H. A. El-Asrag, Y. Ju, Combust. Flame 161 (2014) 256-269.

[24] M. B. Luong, Z. Luo, T. Lu, S. H. Chung, C. S. Yoo, Combust. Flame 160 (2013) 2038-2047.

[25] S. M. Walton, X. He, B. T. Zigler, M. S. Wooldridge, A. Atreya, Combust. Flame 150 (2007) 246-262. 
[26] A. Hultqvist, M. Christenson, B. Johansson, M. Richter, J. Nygren, J. Hult, M. Alden, SAE Technical Paper 2002-01-0424 (2002).

[27] J. E. Dec, W. Hwang, M. Sjoberg, SAE Technical Paper 2006-01-1518 (2006).

[28] J. Krasselt, D. Foster, J. Ghandhi, R. Herold, D. Reuss, P. Najt, SAE Technical Paper 2009-01-1105 (2009).

[29] R. E. Herold, J. M. Krasselt, D. E. Foster, J. B. Ghandhi, SAE Technical Paper 2009-01-1106 (2009).

[30] Y. B. Zeldovich, Combust. Flame 39 (1980) 211-214.

[31] Y. Ju, W. Sun, M. P. Burke, X. Gou, Z. Chen, Proc. Combust. Inst. 33 (2011) $1245-1251$.

[32] A. Bhagatwala, T. Lu, H. Shen, J. A. Sutton, J. H. Chen, Proc. Combust. Inst. (2014). Submitted.

[33] C. A. Kennedy, M. H. Carpenter, Appl. Num. Math. 14 (1994) 397-433.

[34] C. A. Kennedy, M. H. Carpenter, R. M. Lewis, Appl. Num. Math. 35 (2000) $177-219$.

[35] R. J. Kee, F. M. Rupley, J. A. Miller, Chemkin-II: A Fortran Chemical Kinetics Package for the Analysis of Gas-Phase Chemical Kinetics, Technical Report SAND89-8009B, Sandia National Laboratories, 1991.

[36] R. J. Kee, G. Dixon-Lewis, J. Warnatz, M. E. Coltrin, J. A. Miller, A Fortran Computer Code Package for the Evaluation of Gas-Phase Multicompo- 
nent Transport Properties, Technical Report SAND86-8246, Sandia National Laboratories, 1986.

[37] J. O. Hinze, Turbulence, McGraw-Hill, New York, 1975.

[38] C. K. Law, Combustion Physics, Cambridge University Press, 2006.

[39] N. Peters, Turbulent Combustion, Cambridge University Press, 2000.

[40] J. B. Heywood, Internal Combustion Engine Fundamentals, McGraw-Hill, New York, 1988. 


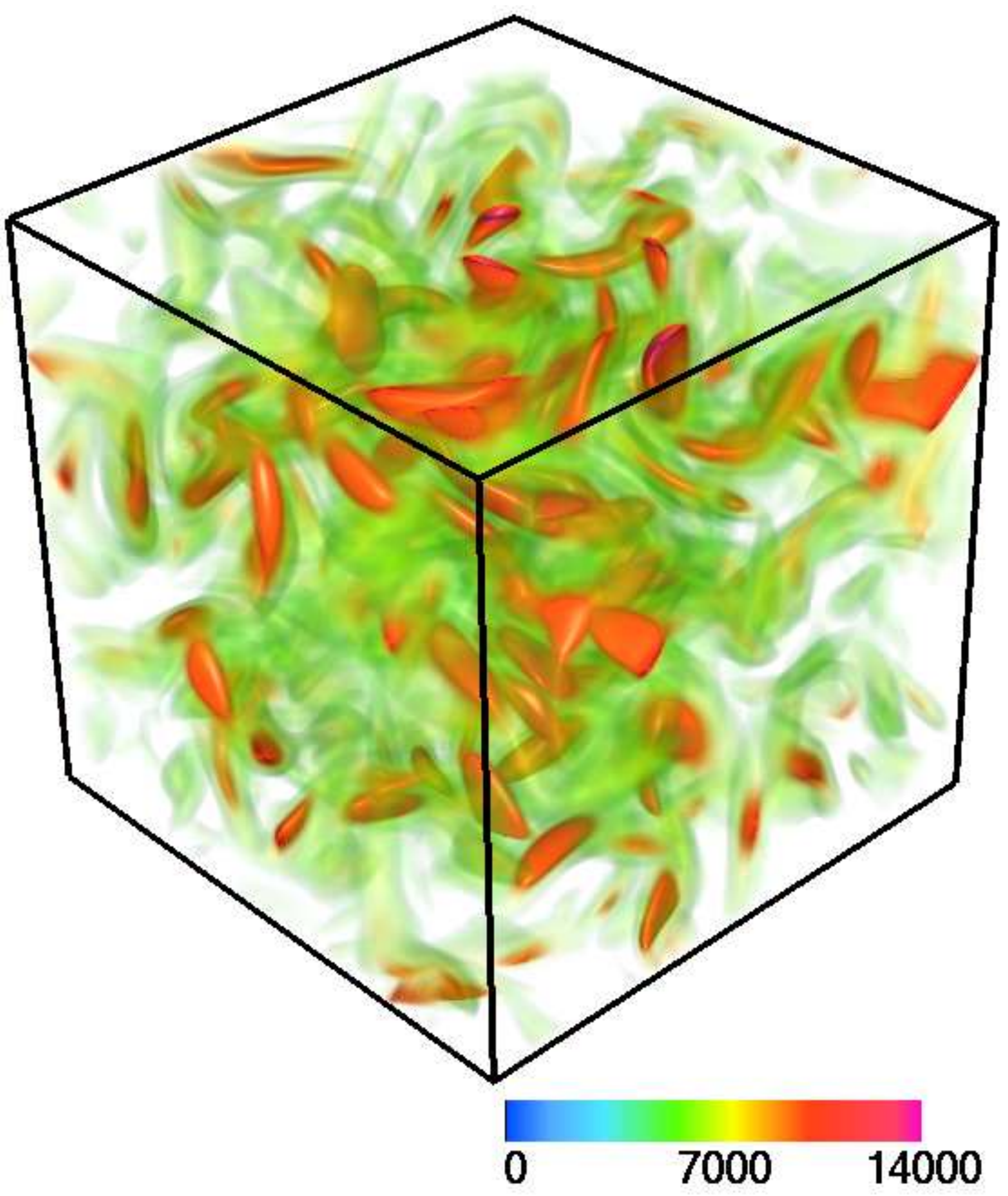




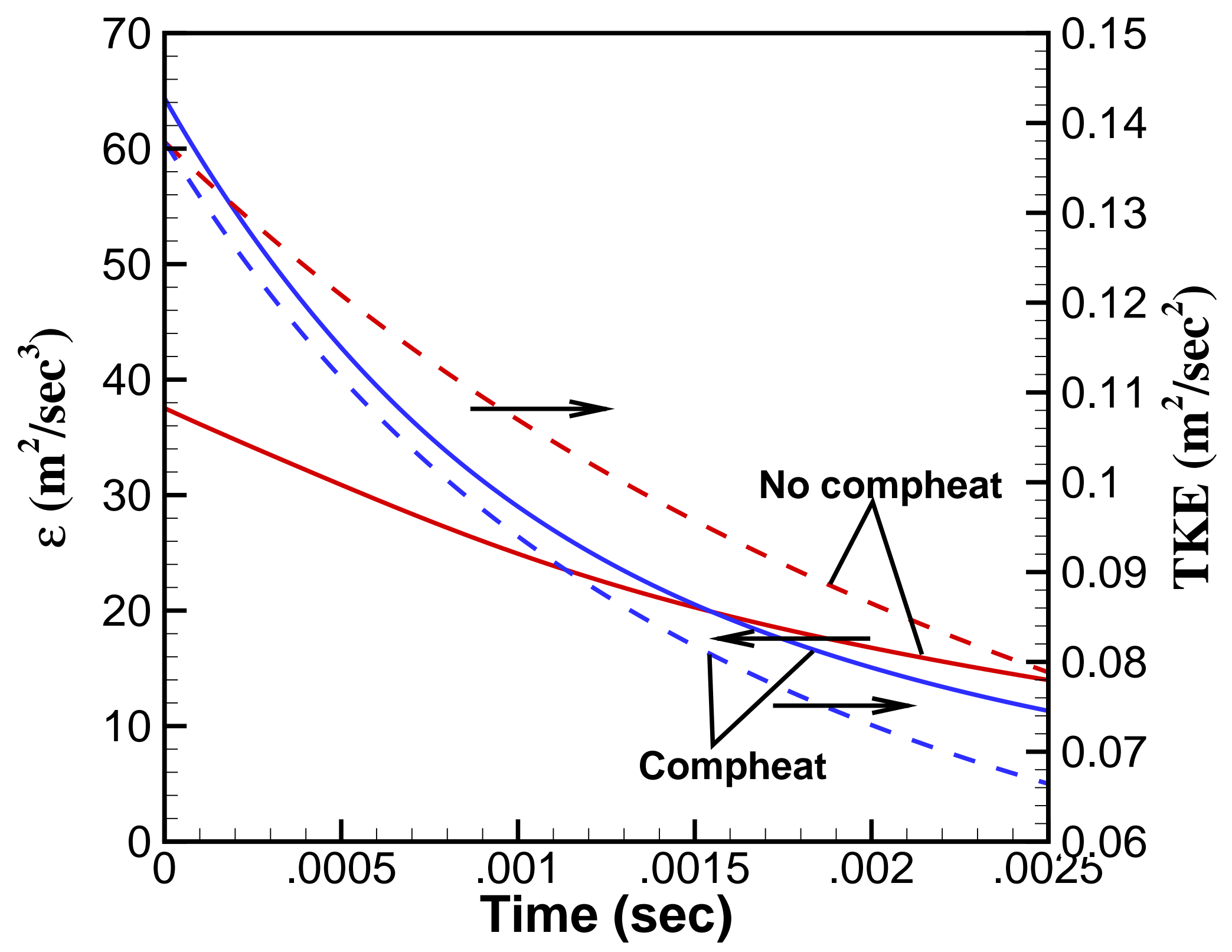




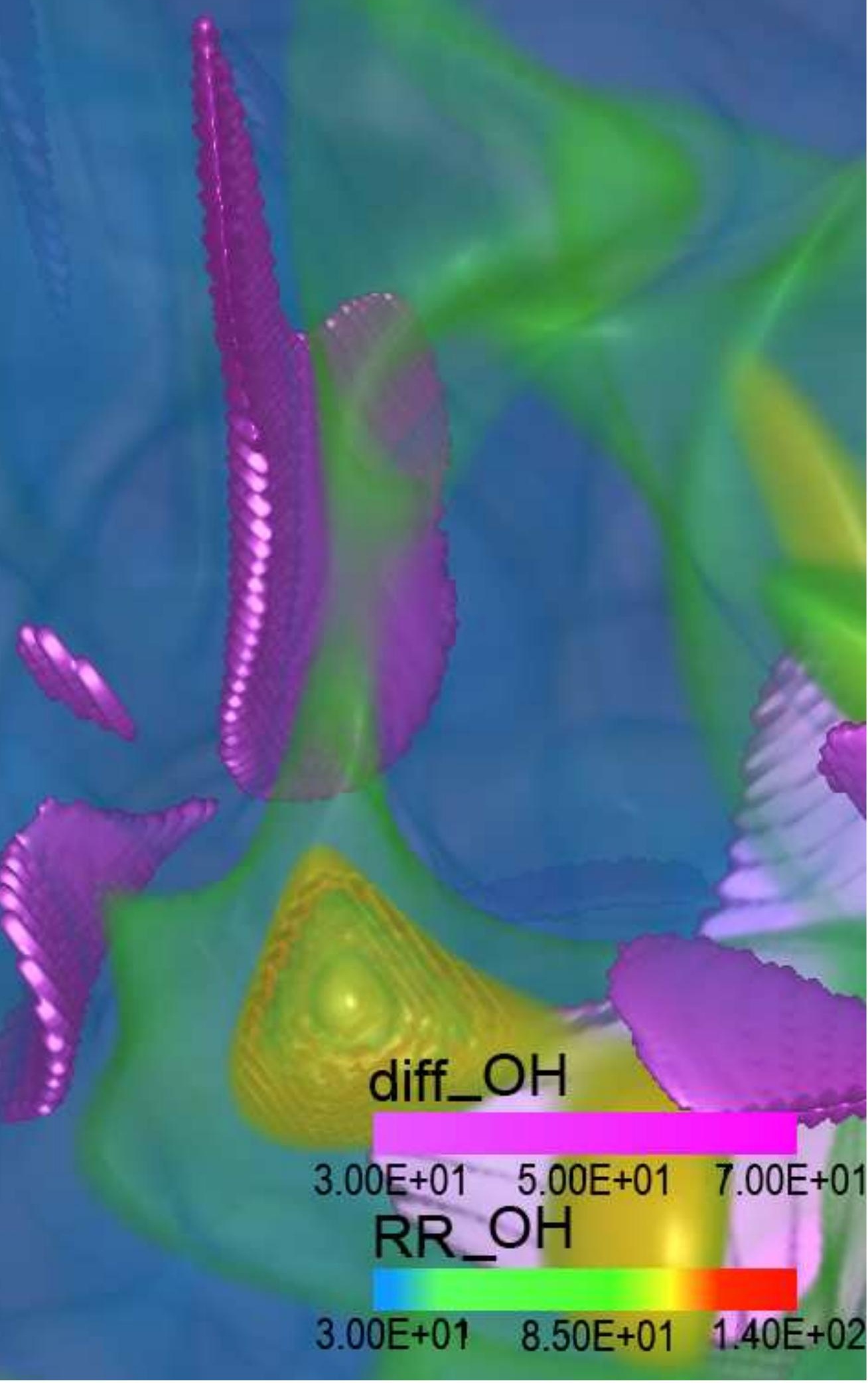

\section{$3.00 E+01 \quad 8.50 E+01 \quad 1.40 E+02$ \\ $3.00 E+01 \quad 5.00 E+01 \quad 7.00 E+01$ $\mathrm{RR} \_\mathrm{OH}$}
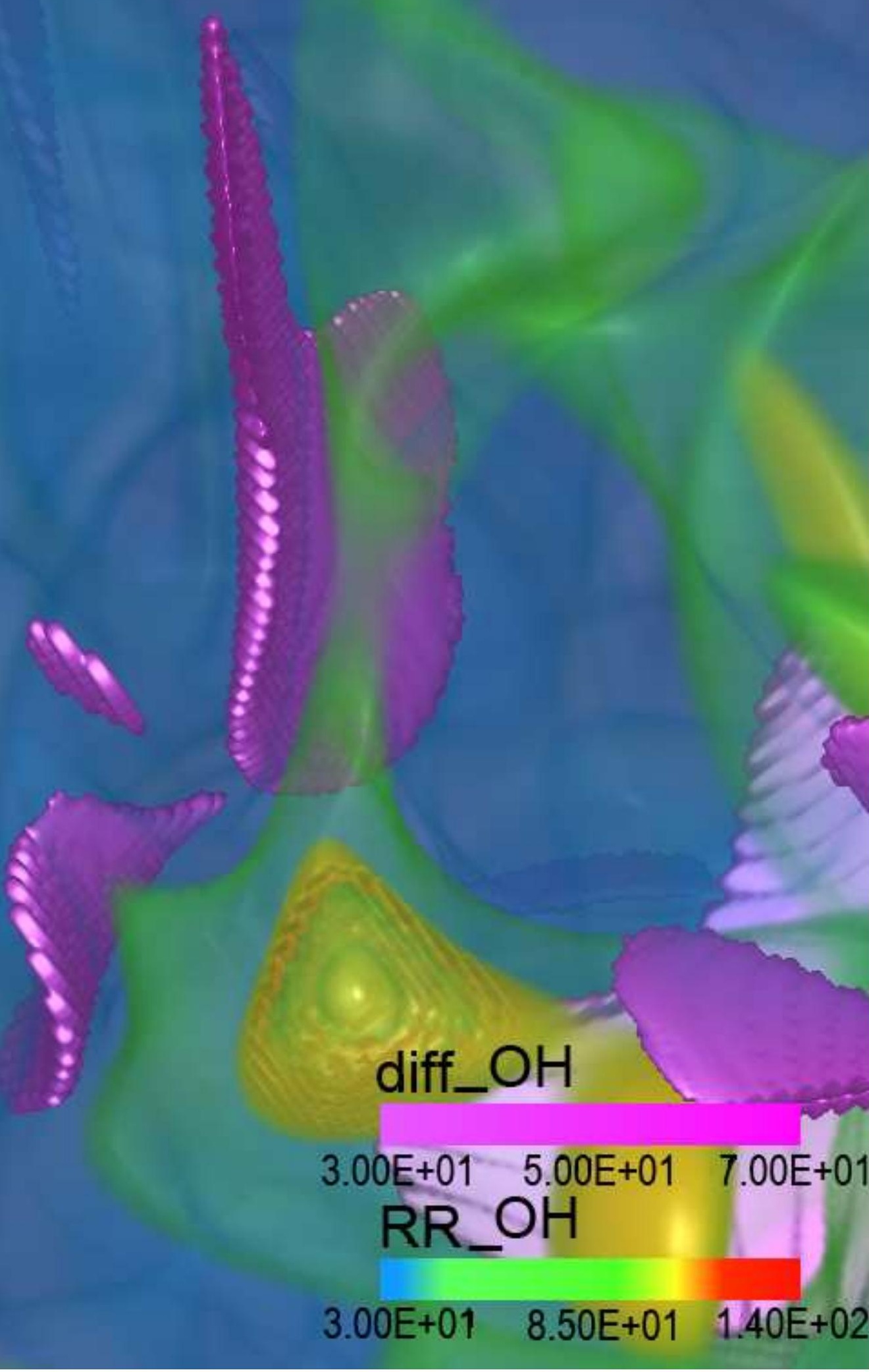


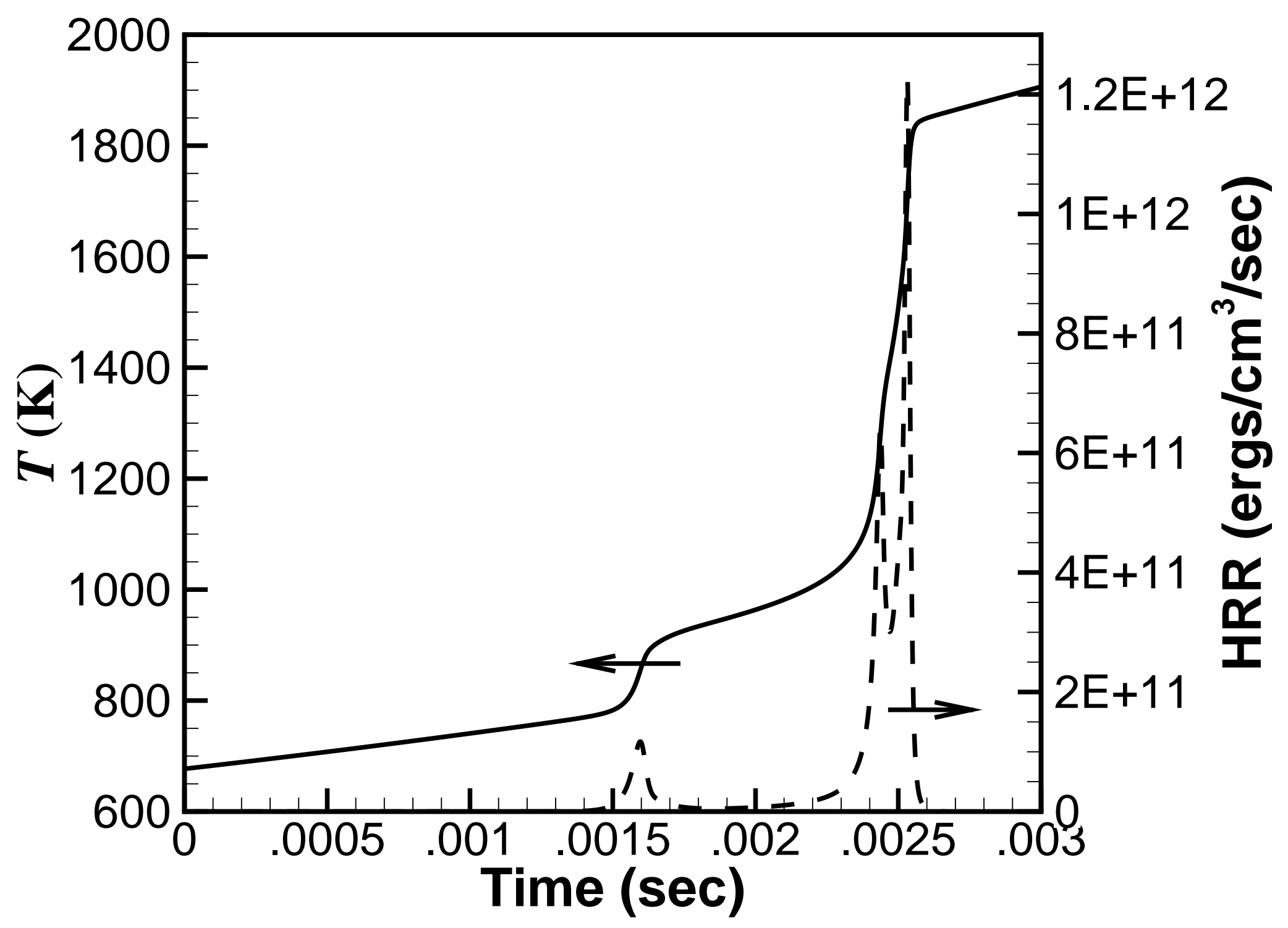




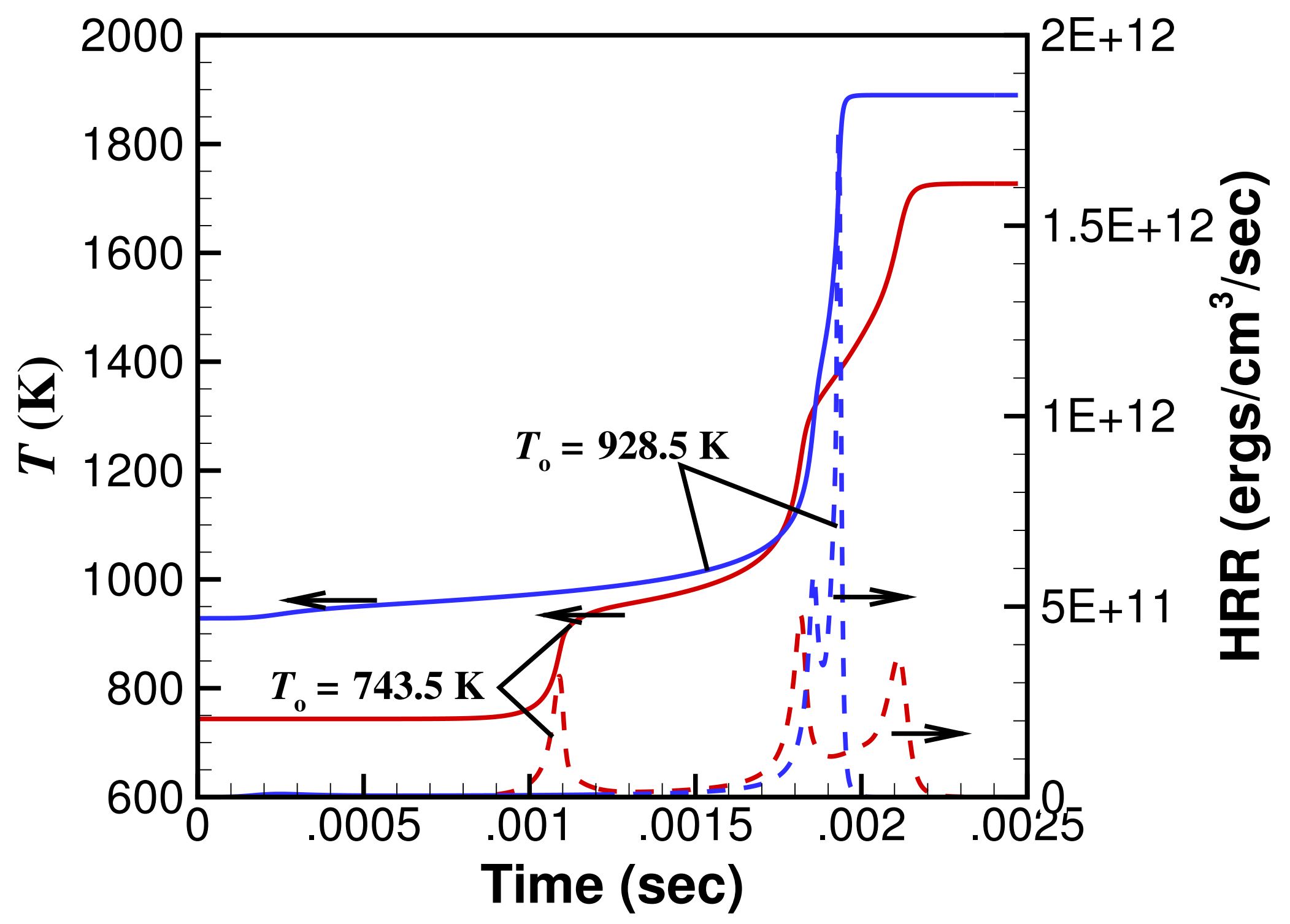




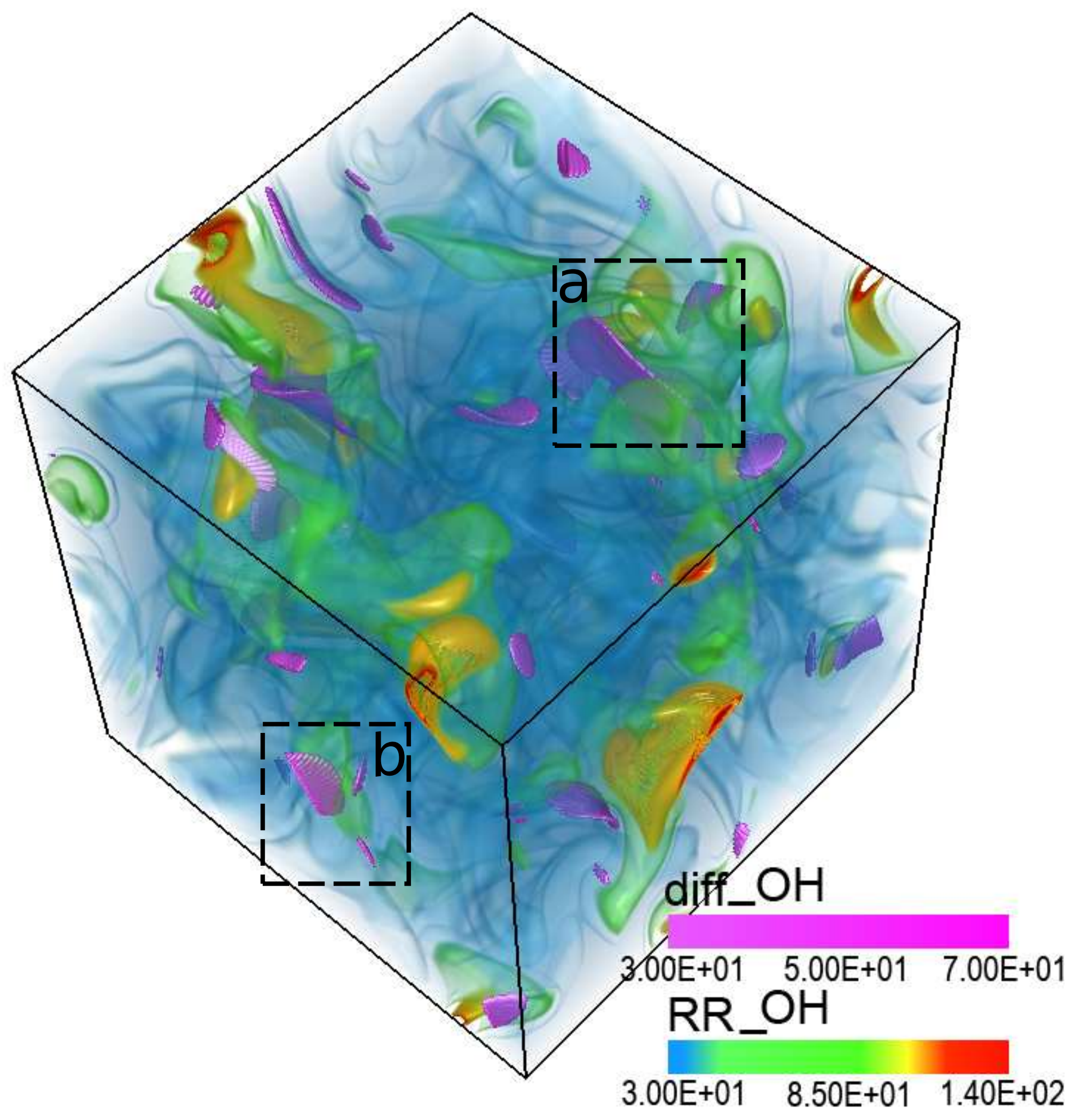




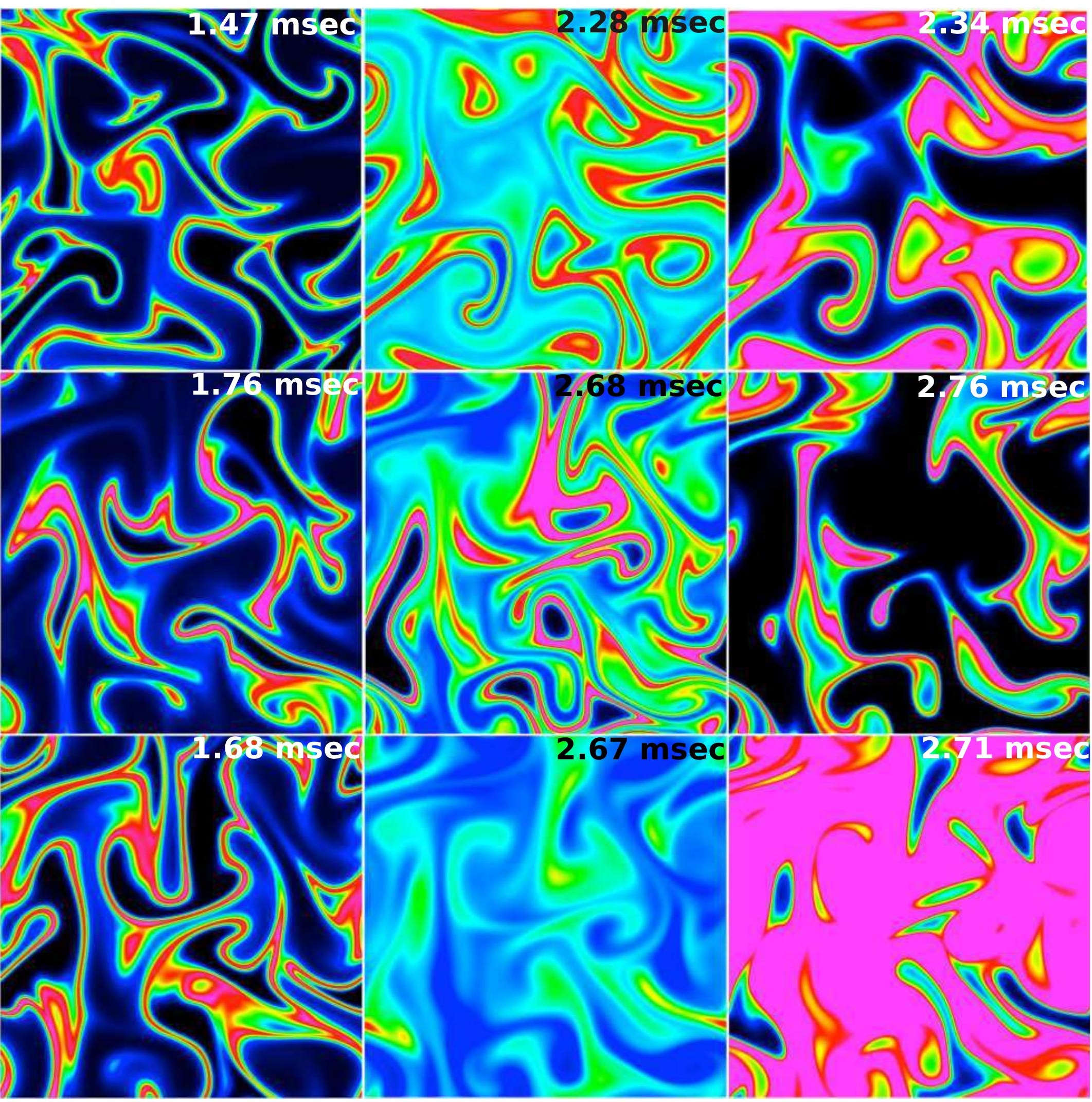




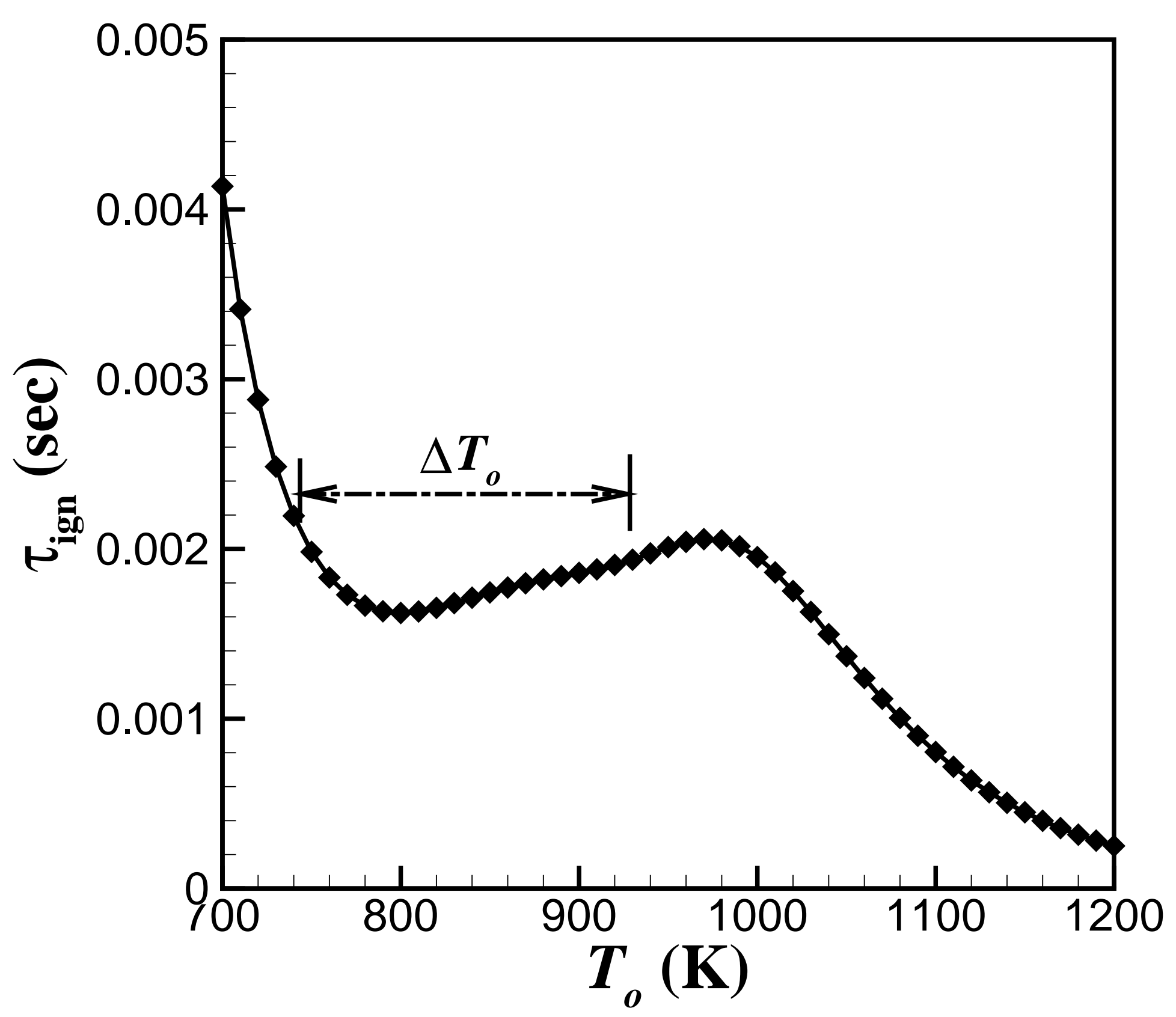




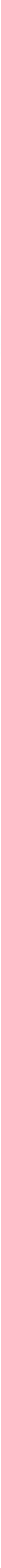

588 


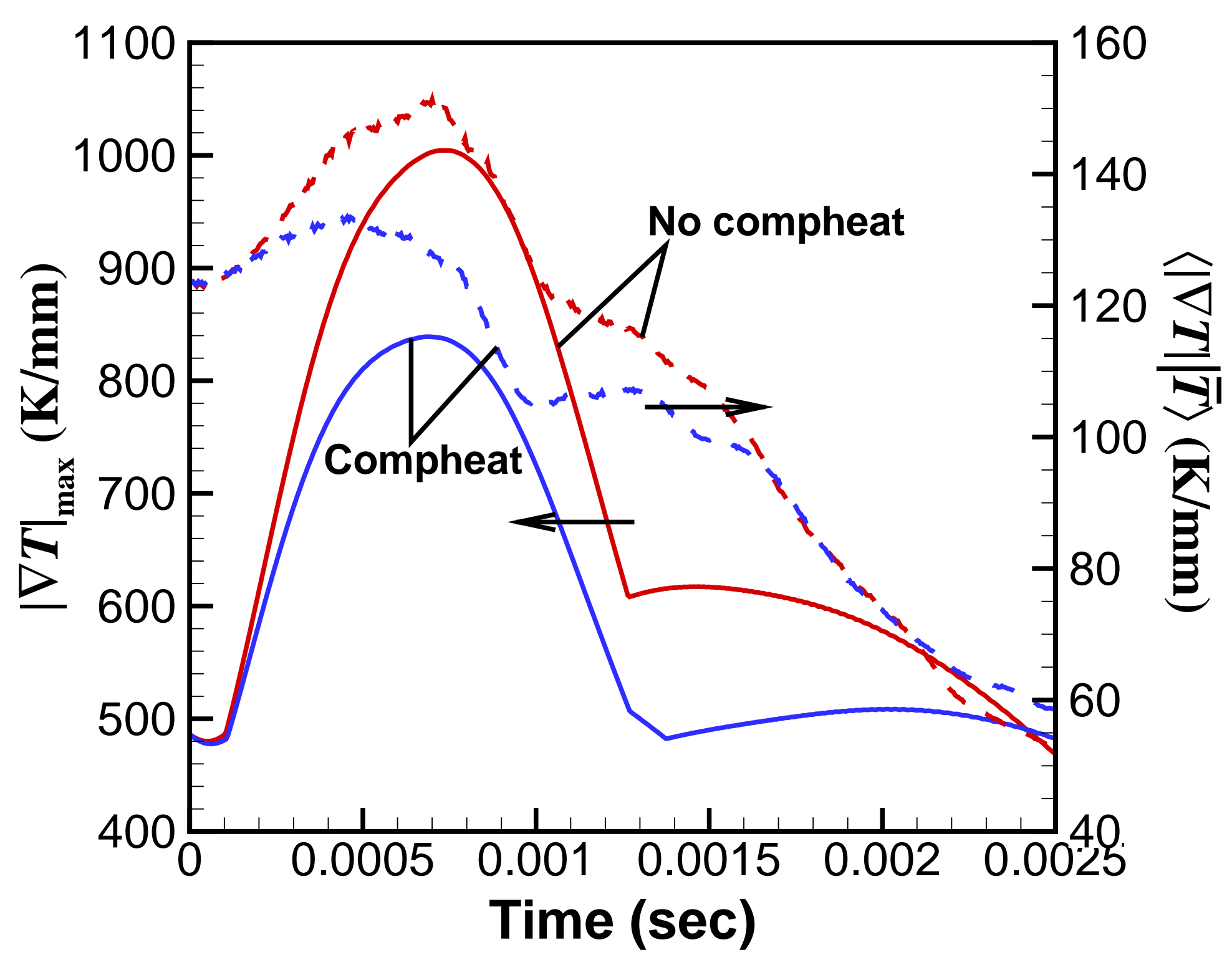




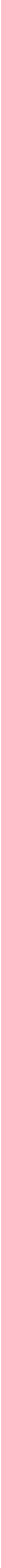

$3.5 e-65$

3e-05

$2.5 e-65$

$2 e-05$

$.5 e-05$

1e-05

5e-06

Tenperature (K) 


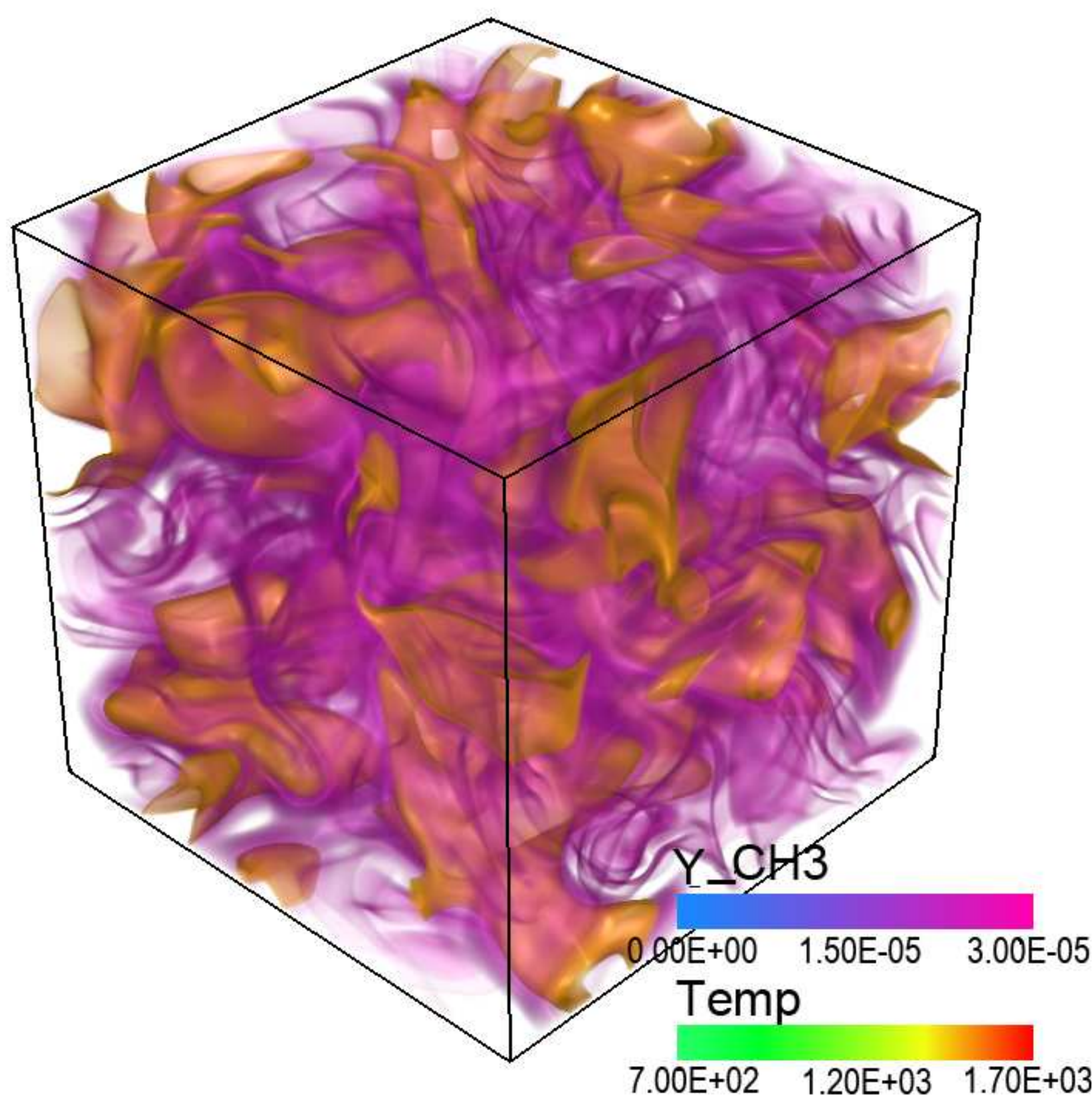




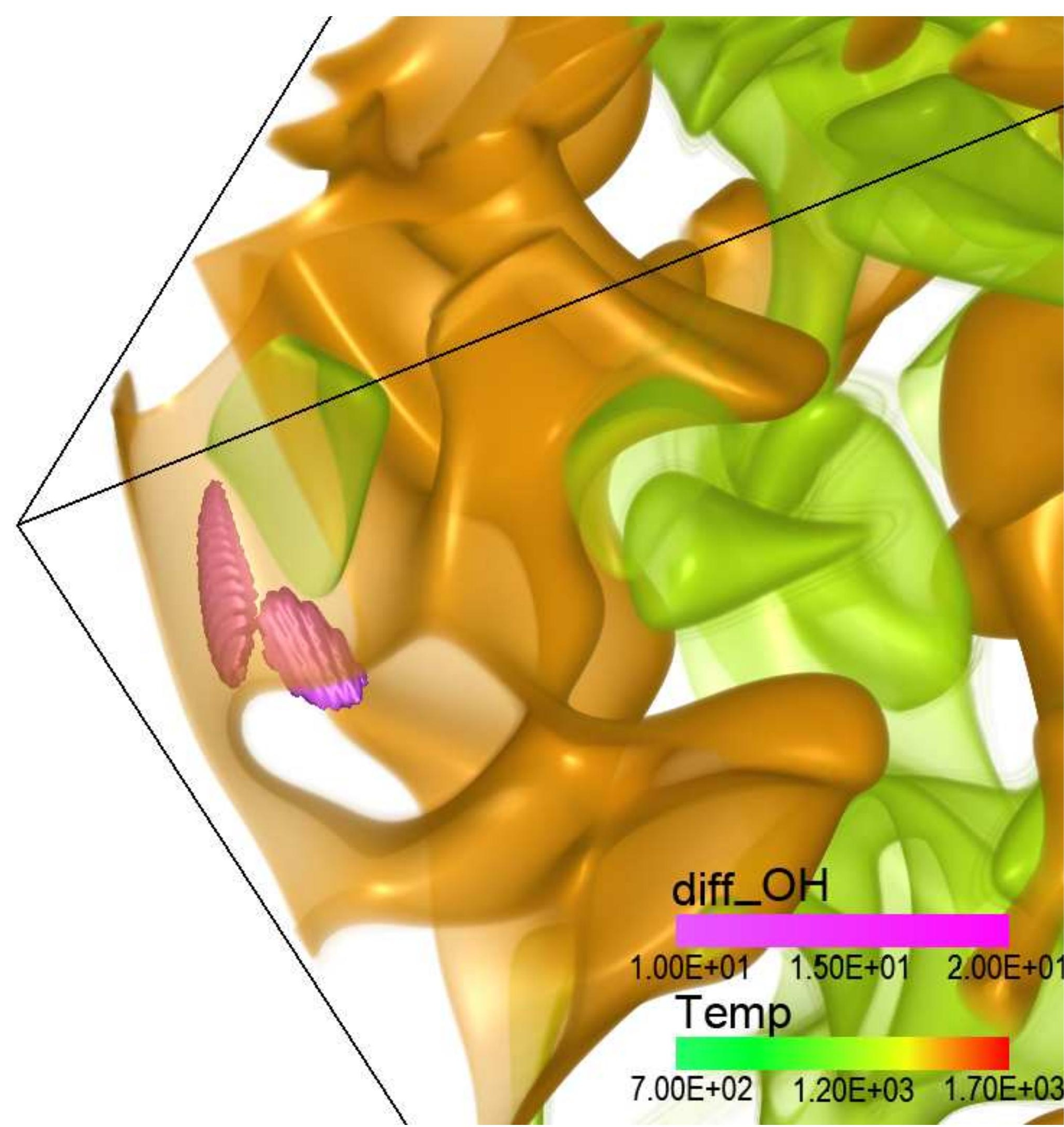




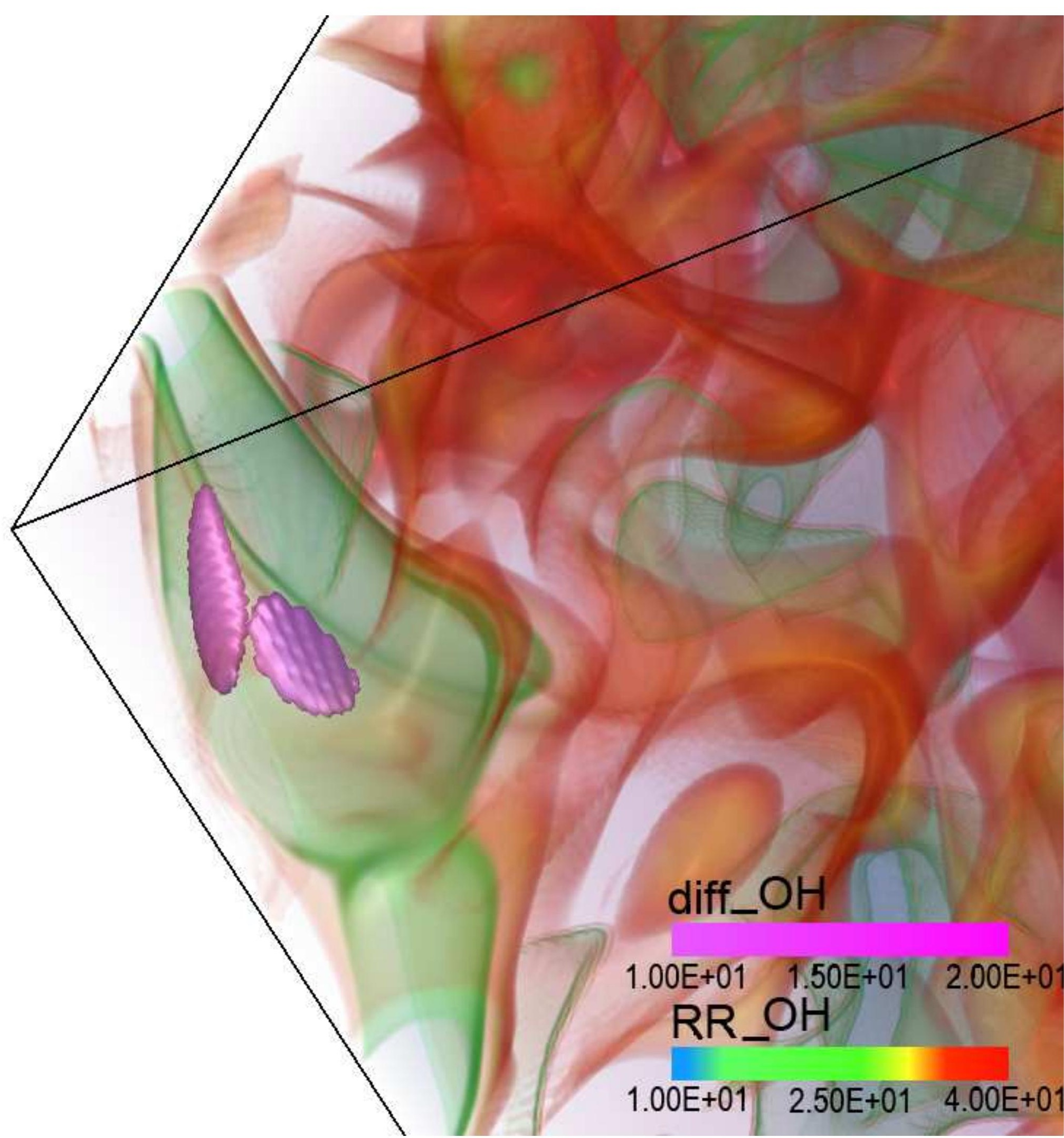




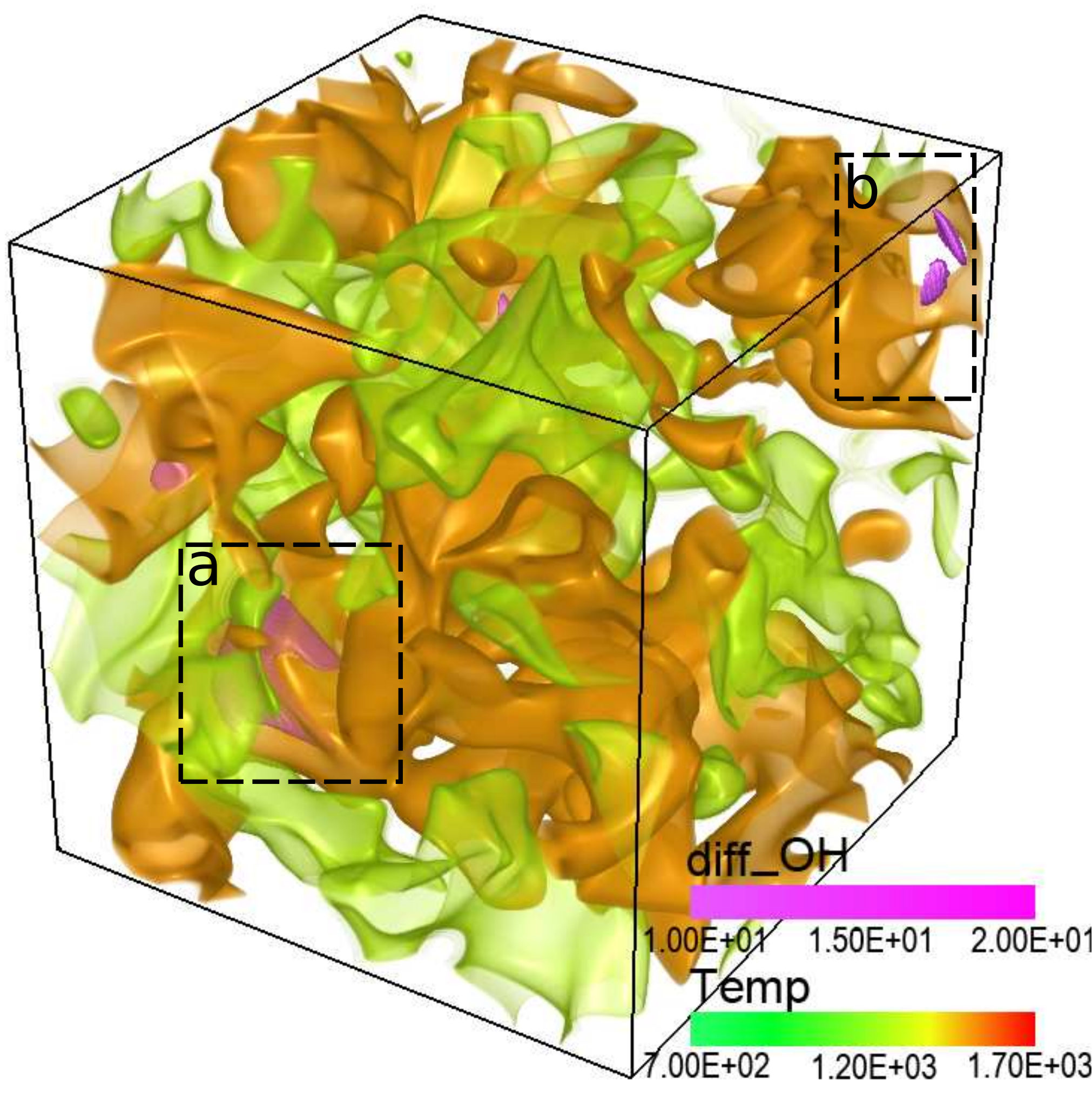




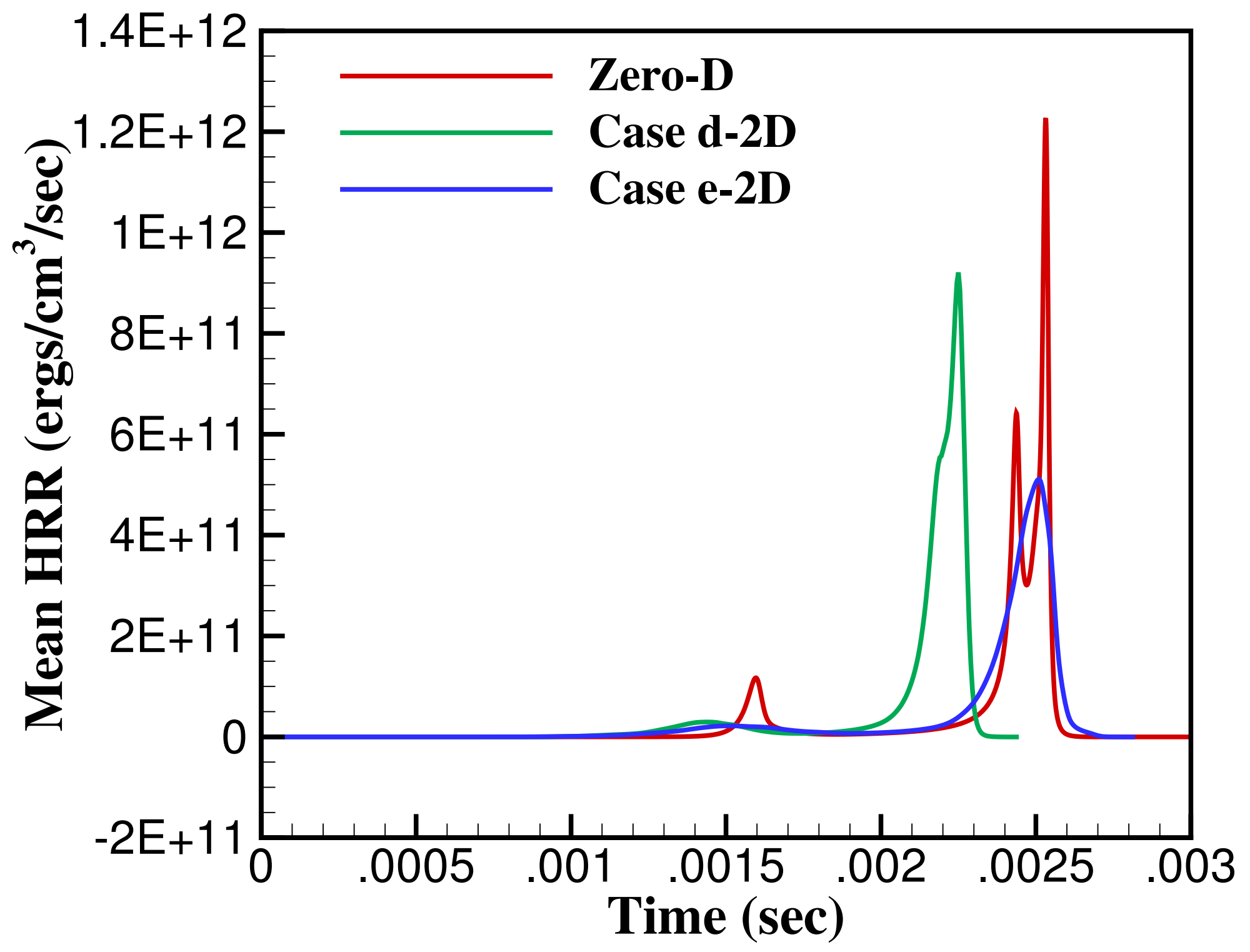




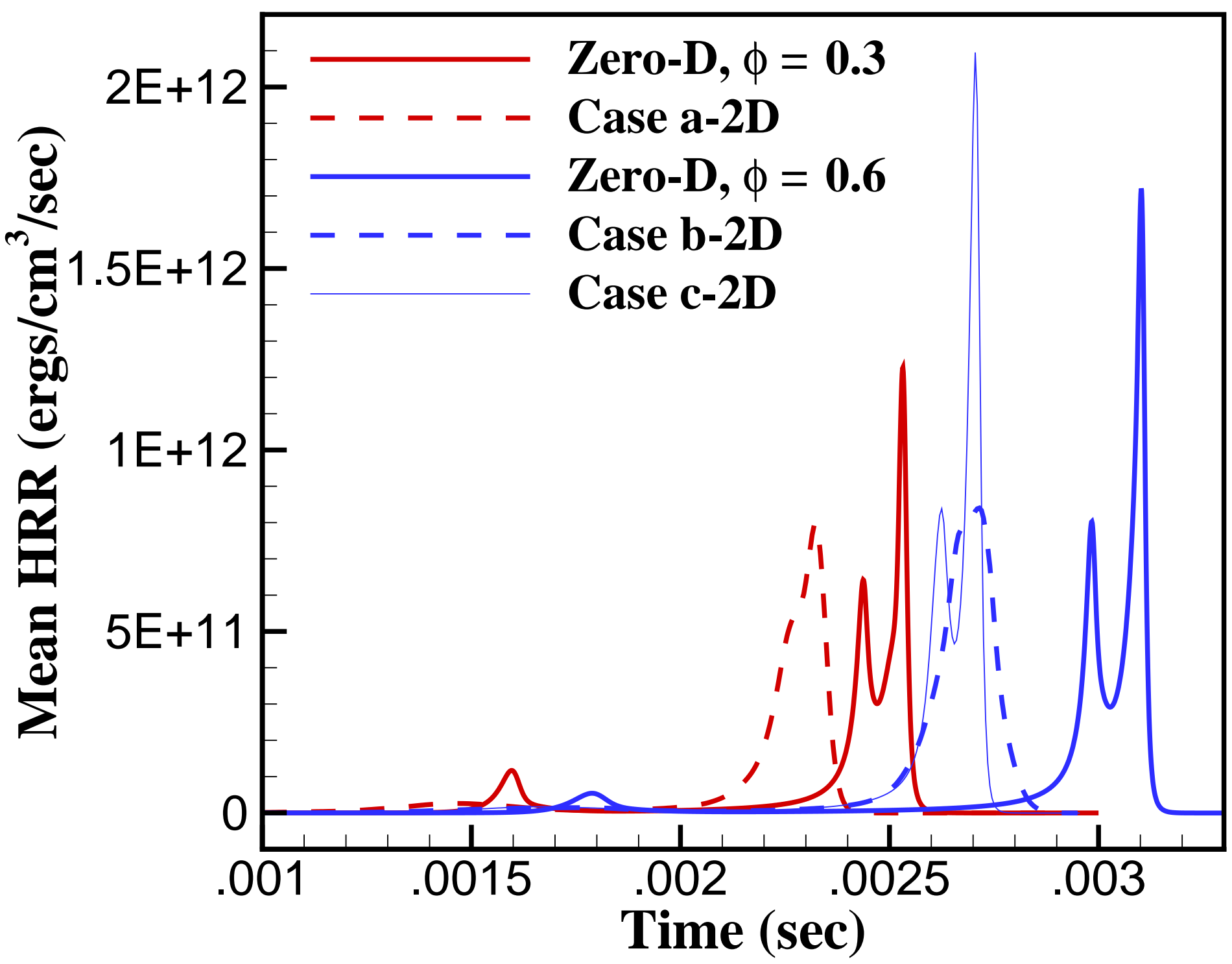




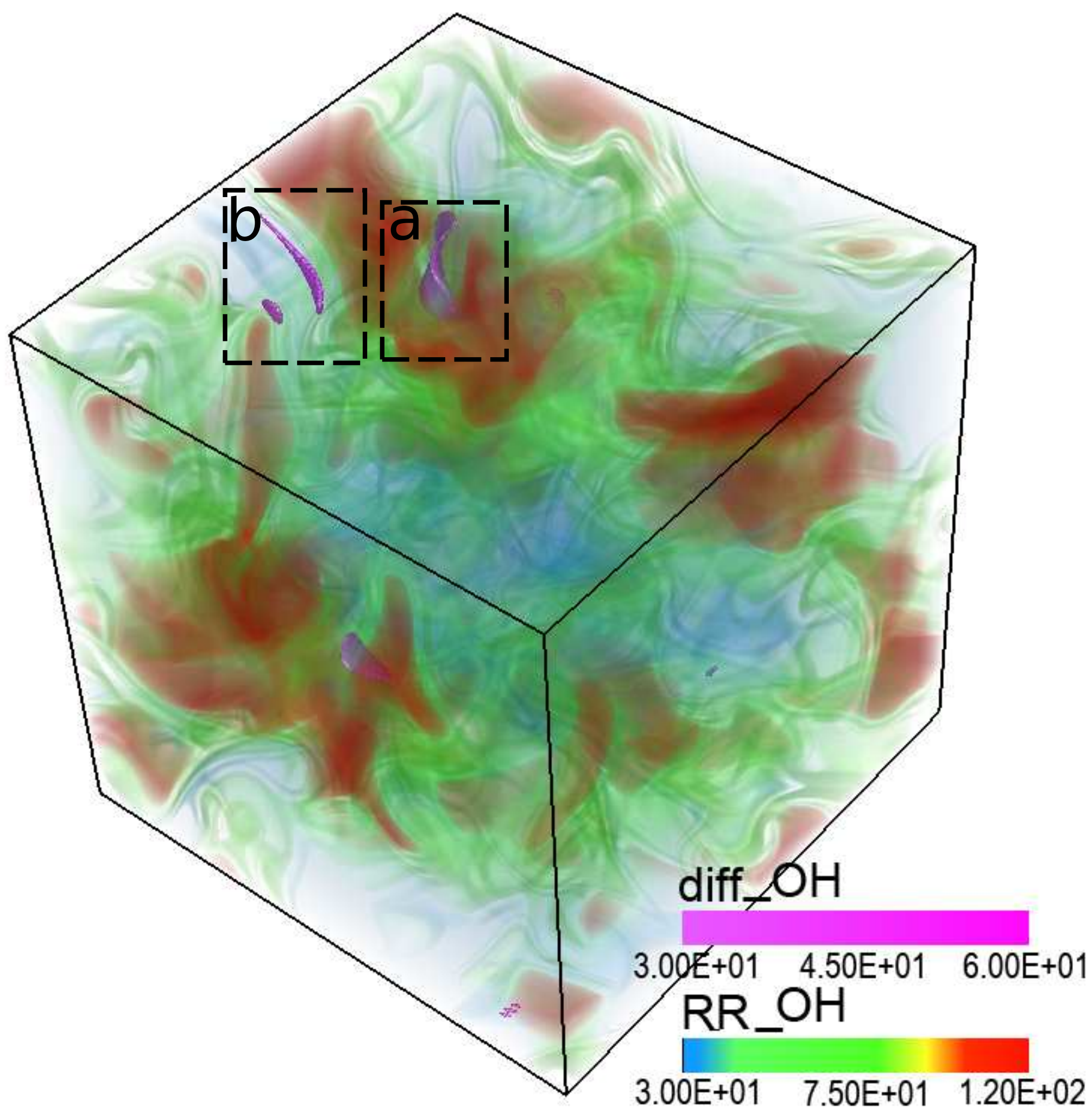




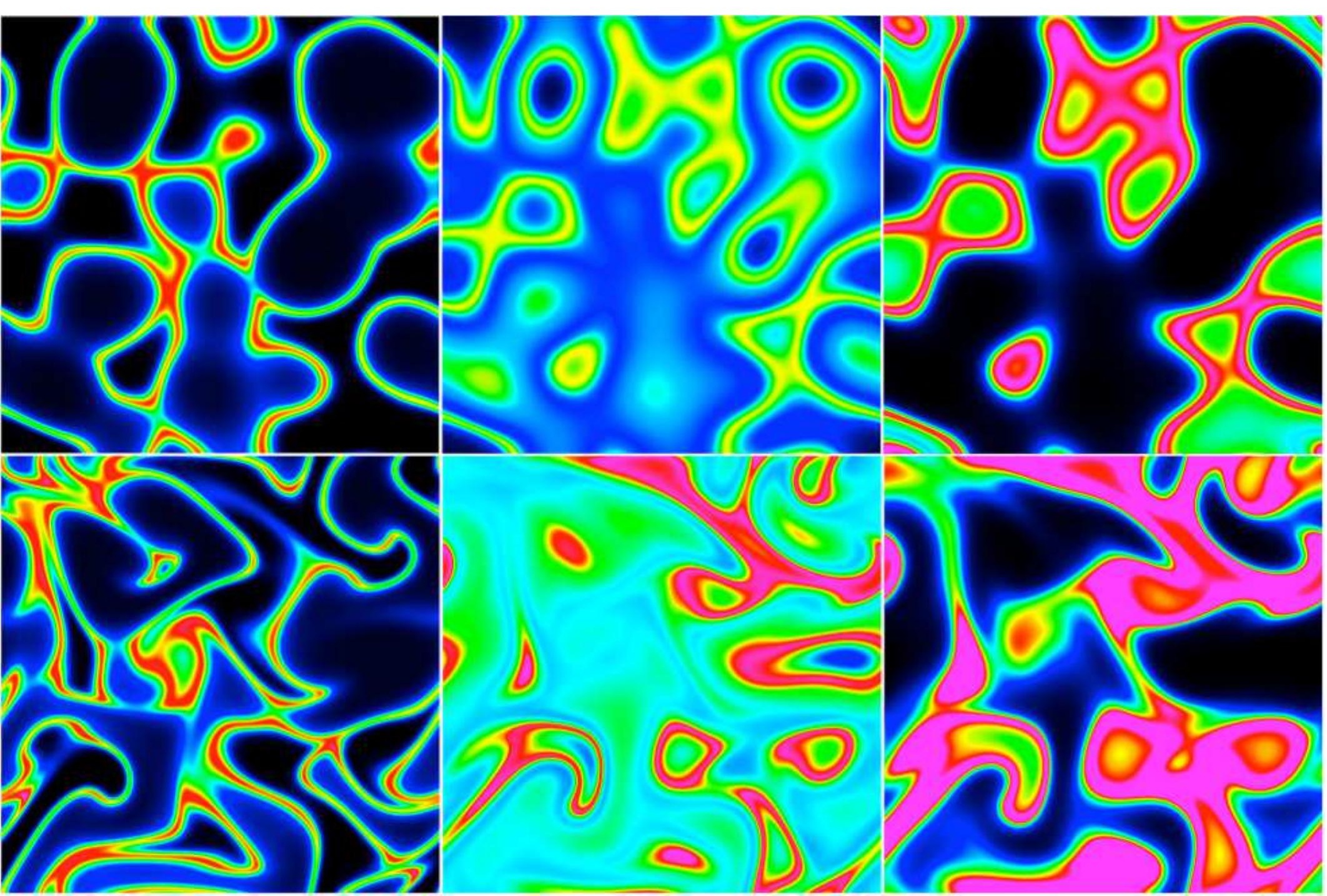




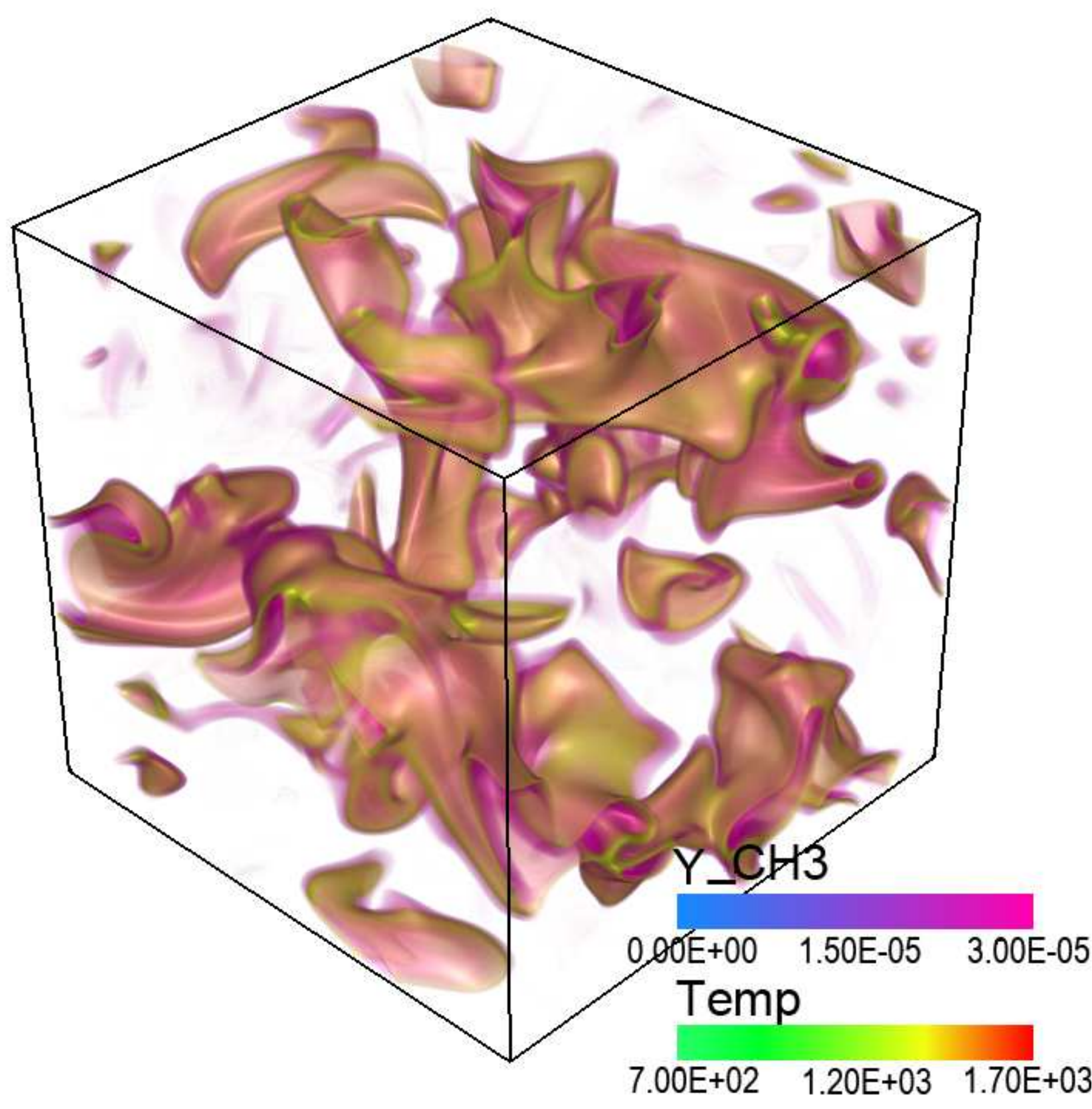




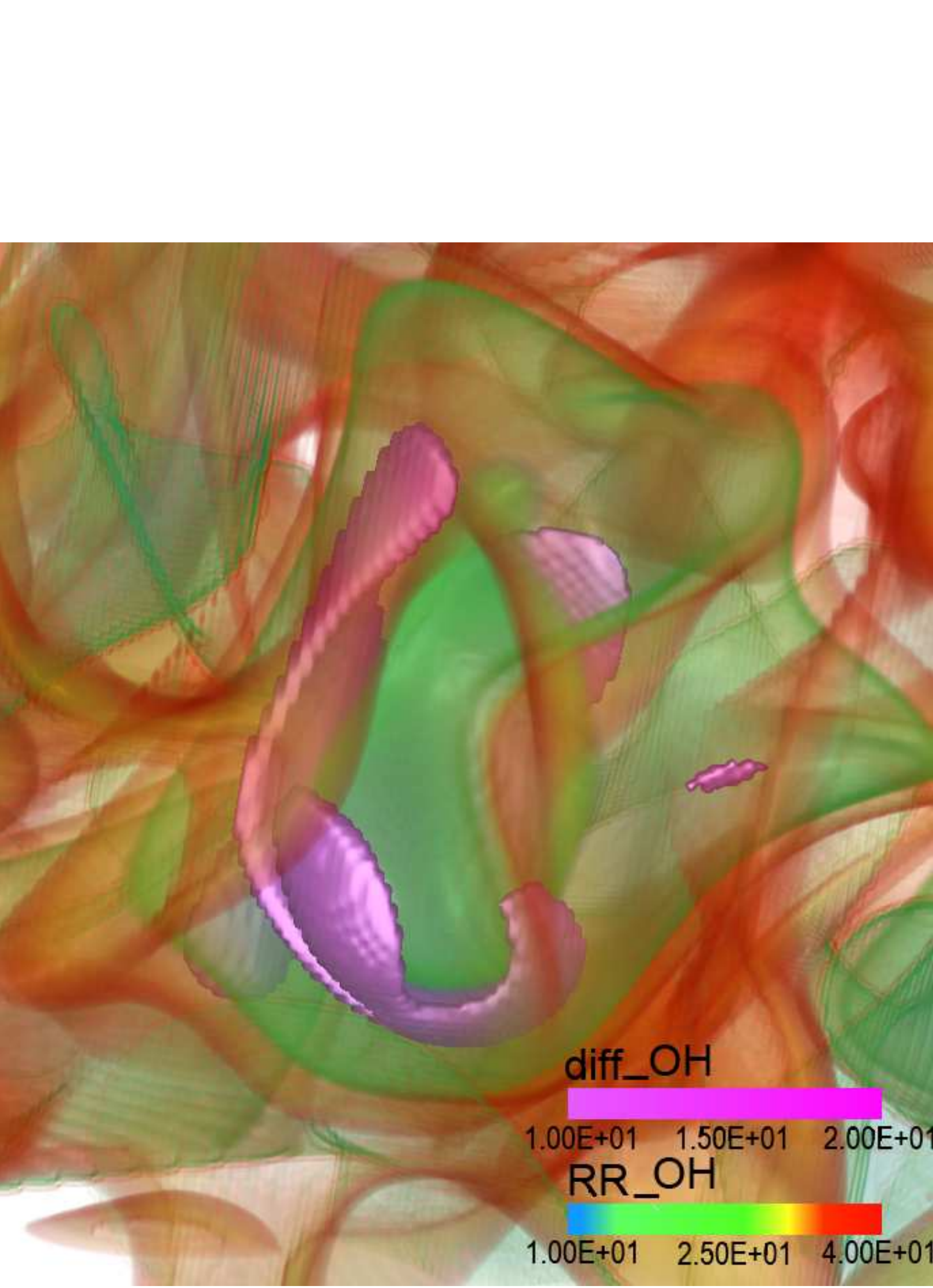




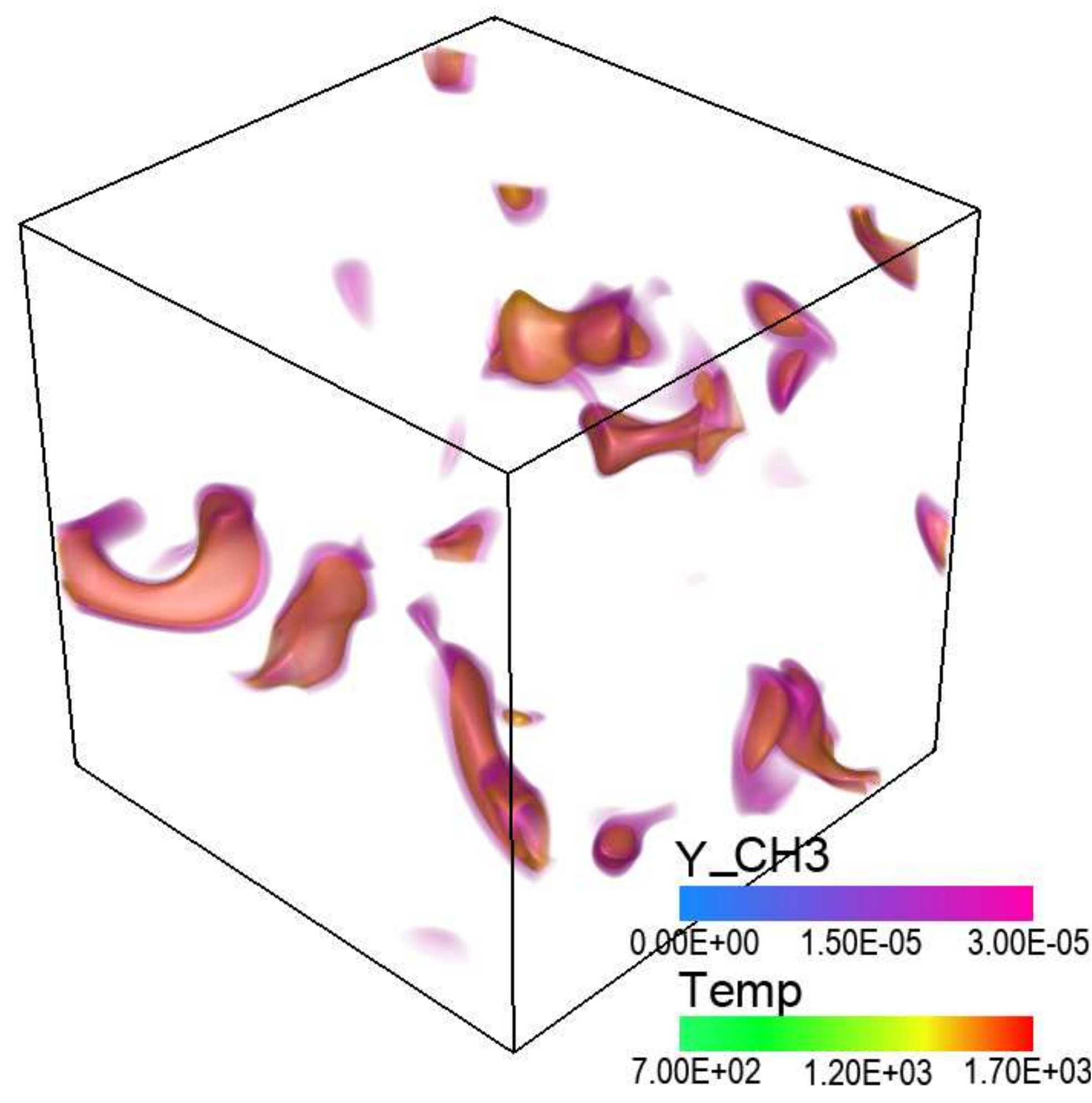




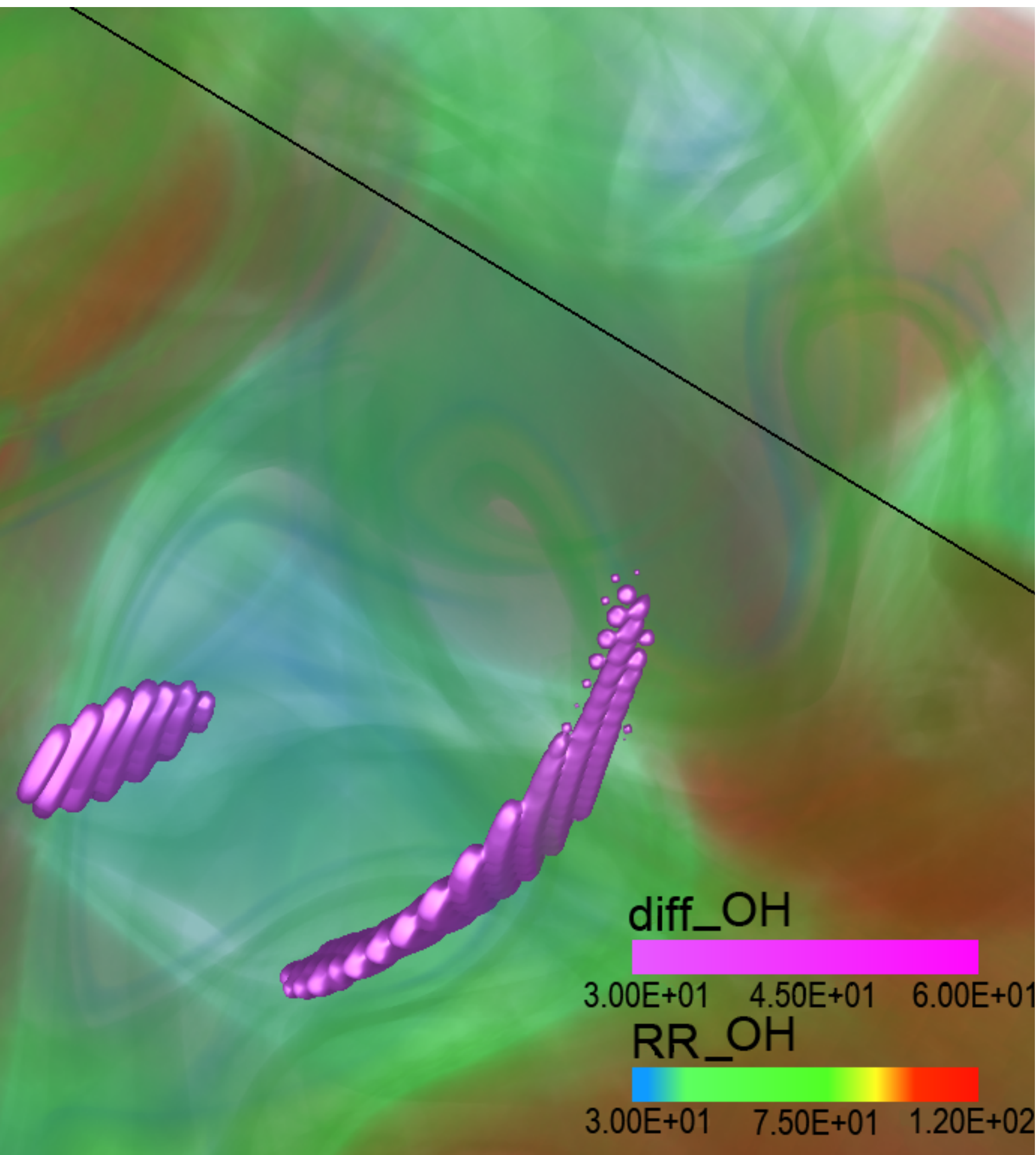

\section{$3.00 \mathrm{E}+01 \quad 4.50 \mathrm{E}+01 \quad 6.00 \mathrm{E}+01$ RR_OH

\section{diff_OH}
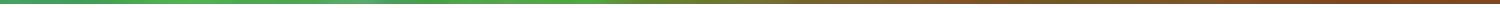


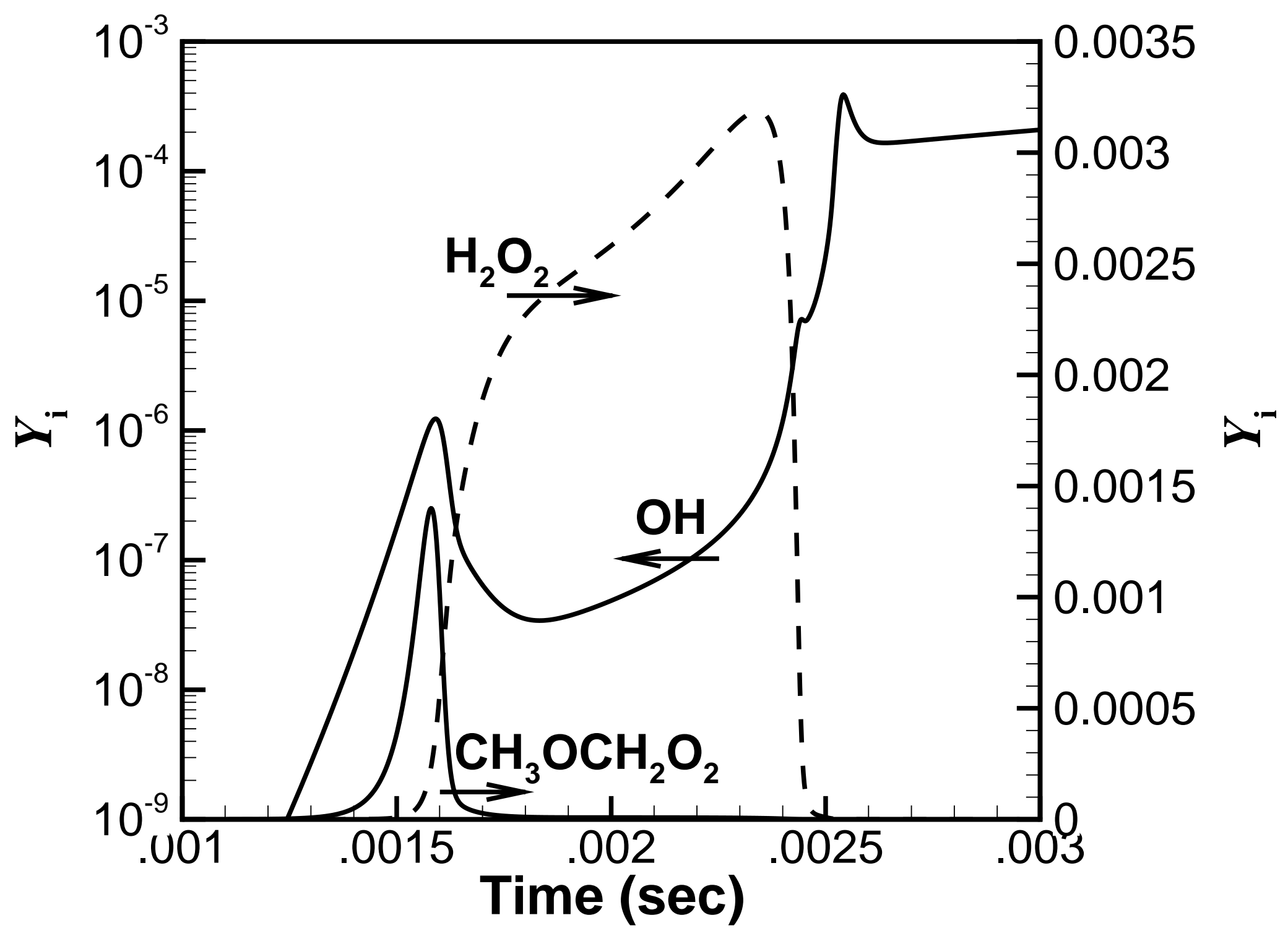




\section{diff_OH}

$1.00 E+01 \quad 1.50 E+01 \quad 2.00 E+01$ Temp

$7.00 \mathrm{E}+02 \quad 1.20 \mathrm{E}+03 \quad 1.70 \mathrm{E}+03$

$7.00 E+02 \quad 1.20 E+03 \quad 1.70 E+03$



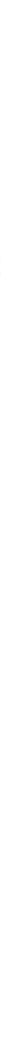

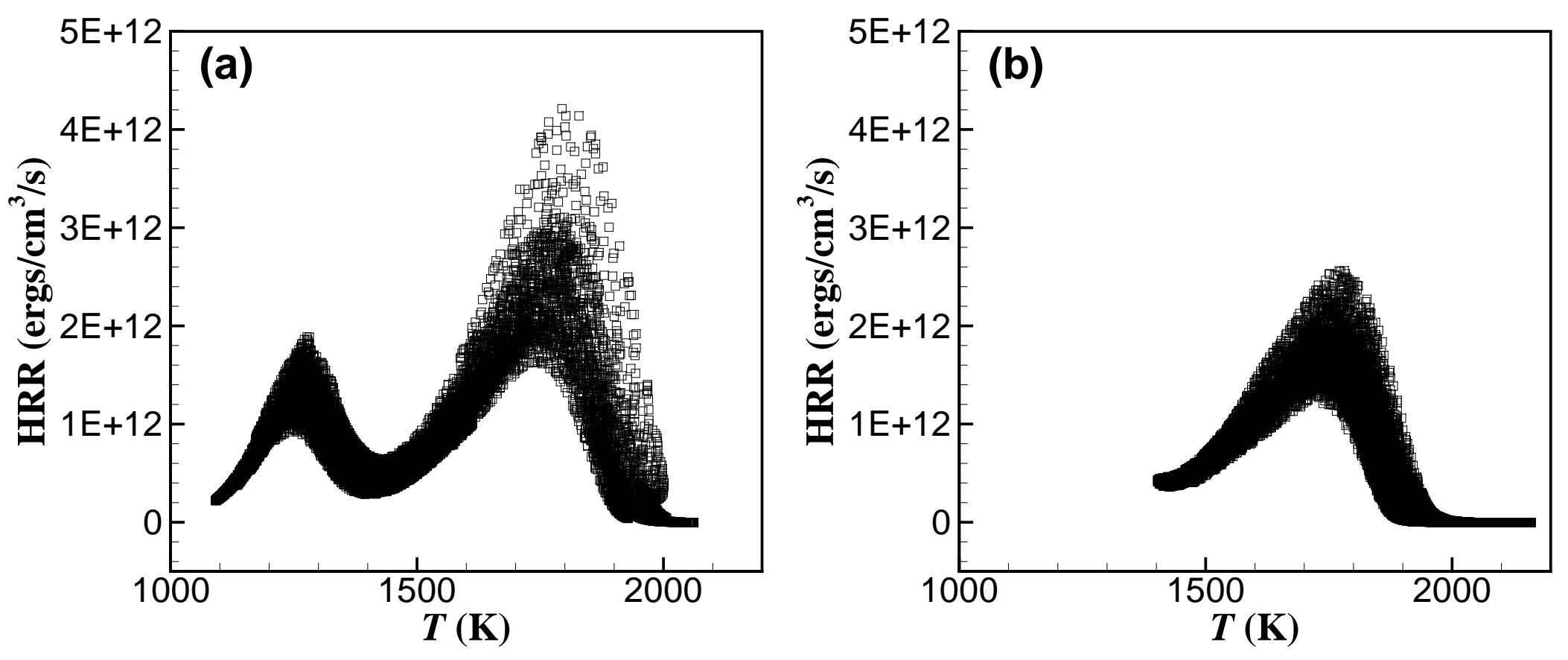


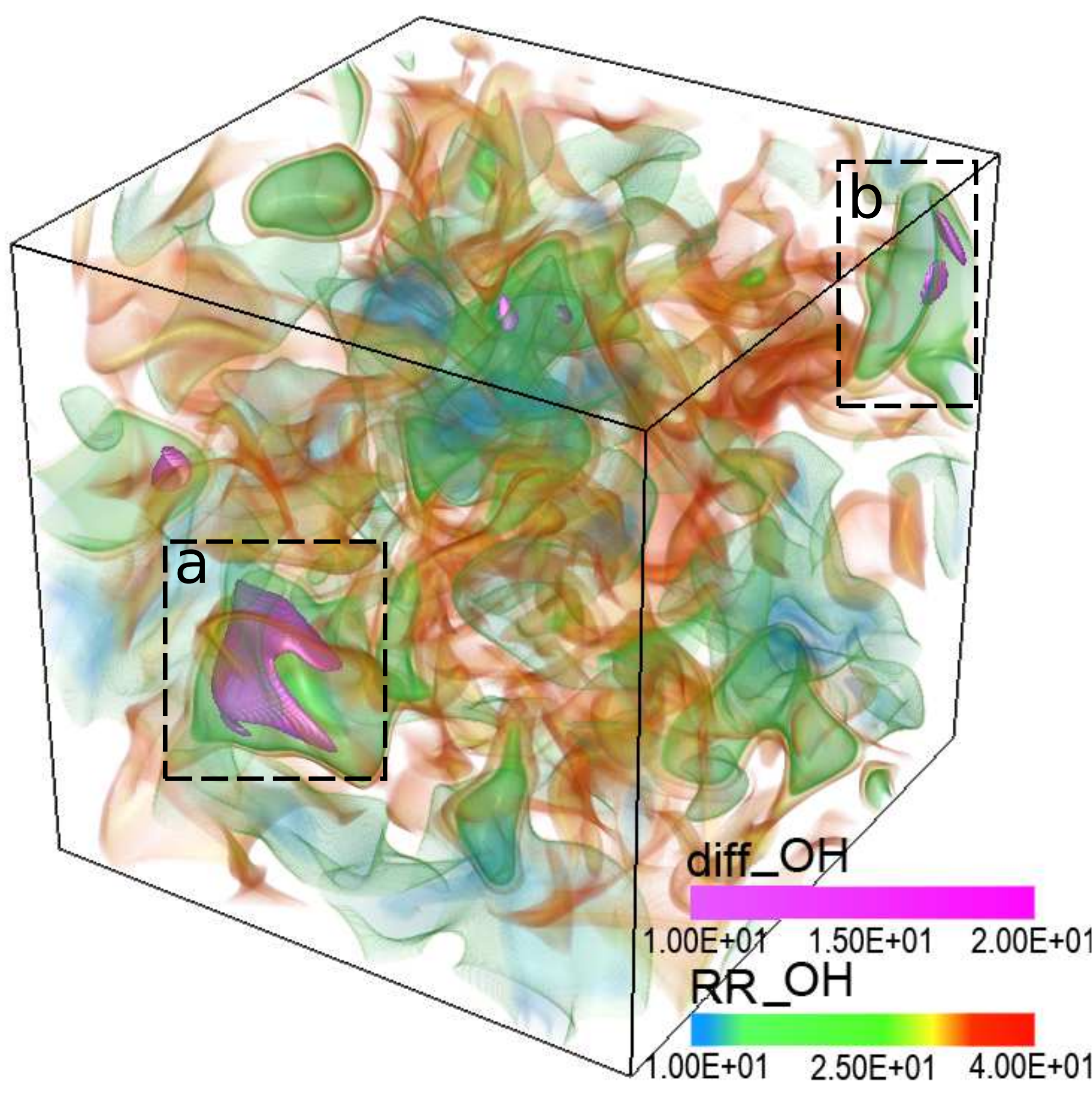


diff_oh_rr_oh_2_1750_view_zoom_1.eps

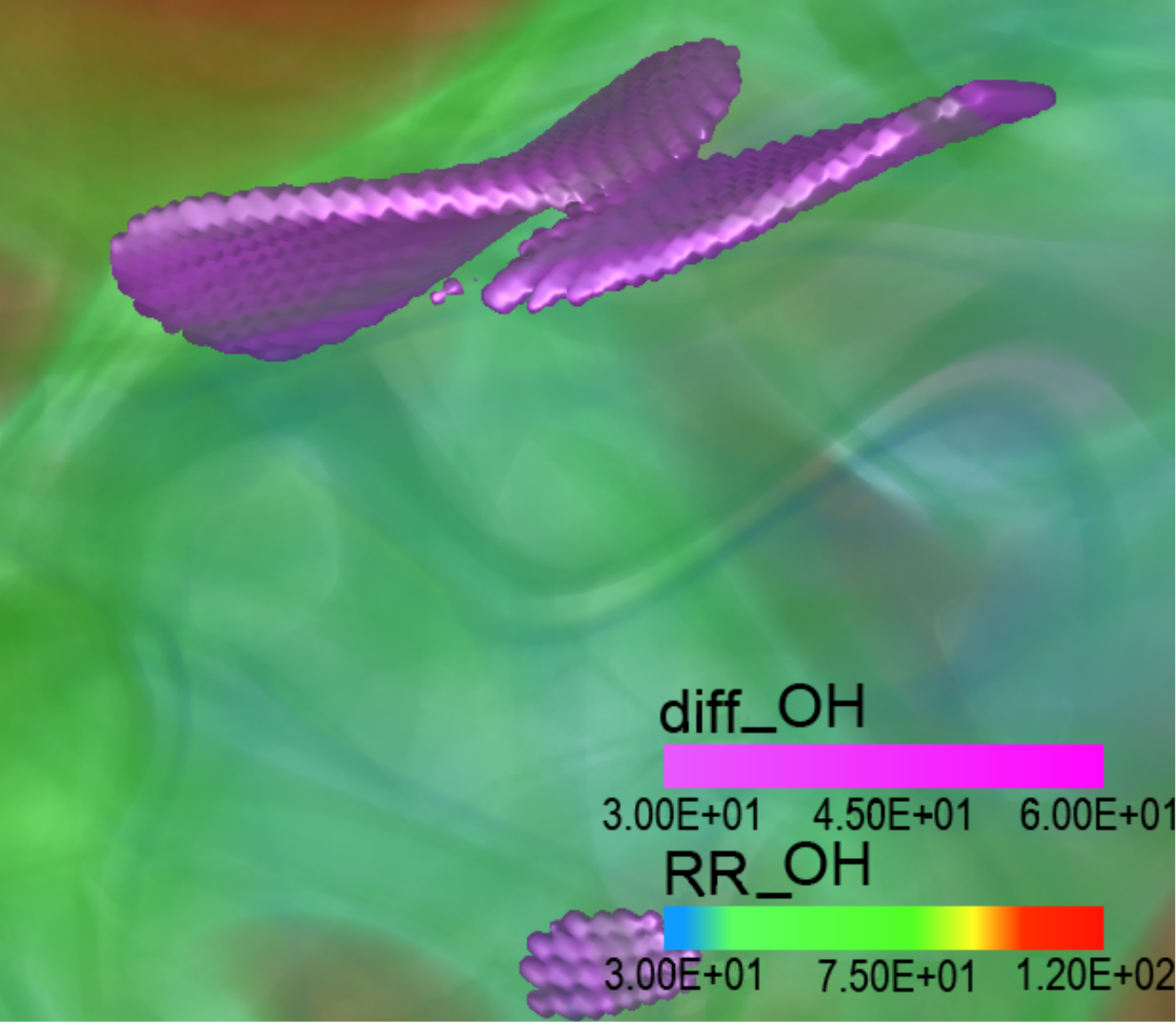

\section{$3.00 \mathrm{E}+01 \quad 4.50 \mathrm{E}+01 \quad 6.00 \mathrm{E}+01$ $\mathrm{RR} \mathrm{OH}$

2007)

diff_OH 


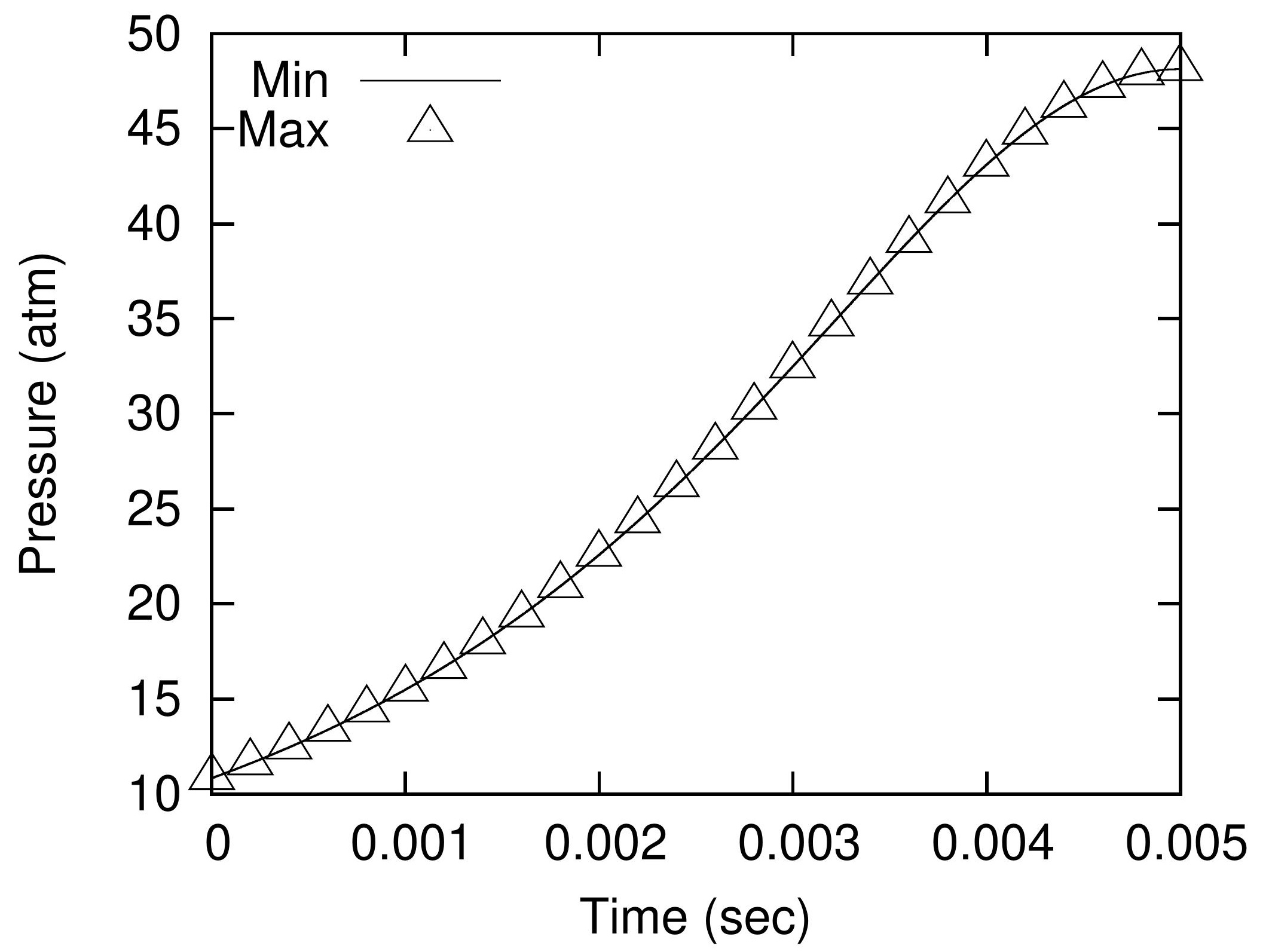




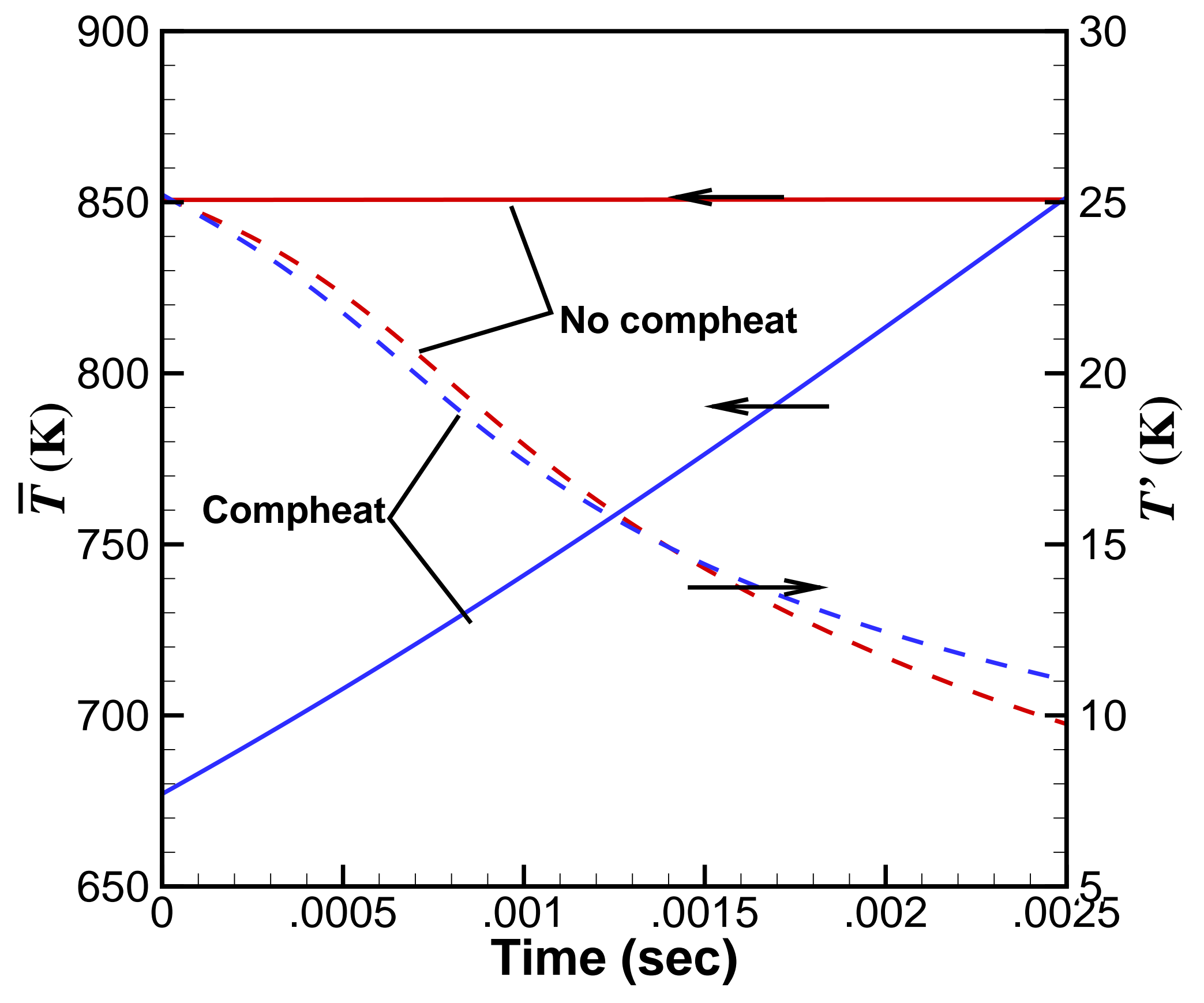

